\title{
Responses of the Housefly, Musca domestica, to the Hytrosavirus Replication: Impacts on Host's Vitellogenesis and Immunity
}

\author{
Henry M. Kariithi ${ }^{1,2}, \mathrm{Xu} \mathrm{Yao}^{3}$, Fahong Yu ${ }^{4}$, Peter E. Teal ${ }^{5 t}$, Chelsea P. Verhoeven ${ }^{3}$ and \\ Drion G. Boucias ${ }^{3 *}$ \\ ${ }^{1}$ Biotechnology Research Institute, Kenya Agricultural and Livestock Research Organization, Nairobi, Kenya, ${ }^{2}$ Insect Pest \\ Control Laboratory, Joint FAO/IAEA Division of Nuclear Techniques in Food and Agriculture, Vienna, Austria, ${ }^{3}$ Entomology \\ and Nematology Department, University of Florida, Gainesville, FL, USA, ${ }^{4}$ Interdisciplinary Centre for Biotechnology \\ Research, University of Florida, Gainesville, FL, USA, ${ }^{5}$ Center for Medical, Agricultural and Veterinary Entomology, USDA, \\ ARS, Gainesville, FL, USA
}

OPEN ACCESS

Edited by:

Akio Adachi,

Tokushima University, Japan

Reviewed by:

Peter Krell,

University of Guelph, Canada

Gong-yin Ye,

Zhejiang University, China

*Correspondence:

Drion G. Boucias

pathos@ufl.edu

${ }^{\dagger}$ Deceased.

Specialty section:

This article was submitted to

Virology,

a section of the journal

Frontiers in Microbiology

Received: 17 February 2017 Accepted: 21 March 2017

Published: 05 April 2017

Citation:

Kariithi HM, Yao X, YU F, Teal PE, Verhoeven CP and Boucias DG (2017)

Responses of the Housefly, Musca domestica, to the Hytrosavirus Replication: Impacts on Host's Vitellogenesis and Immunity.

Front. Microbiol. 8:583

doi: 10.3389/fmicb.2017.00583
Hytrosaviridae family members replicate in the salivary glands (SGs) of their adult dipteran hosts and are transmitted to uninfected hosts via saliva during feeding. Despite inducing similar gross symptoms (SG hypertrophy; SGH), hytrosaviruses (SGHVs) have distinct pathobiologies, including sex-ratio distortions in tsetse flies and refusal of infected housefly females to copulate. Via unknown mechanism(s), SGHV replication in other tissues results in reduced fecundity in tsetse flies and total shutdown of vitellogenesis and sterility in housefly females. We hypothesized that vitellogenesis shutdown was caused by virus-induced modulation of hormonal titers. Here, we used RNA-Seq to investigate virus-induced modulation of host genes/pathways in healthy and virus-infected houseflies, and we validated expression of modulated genes $(n=$ 23) by RT-qPCR. We also evaluated the levels and activities of hemolymph AMPs, levels of endogenous sesquiterpenoids, and impacts of exogenous hormones on ovarian development in viremic females. Of the 973 housefly unigenes that were significantly modulated (padj $\leq 0.01, \log 2 \mathrm{FC} \leq-2.0$ or $\geq 2.0$ ), 446 and 527 genes were downregulated and upregulated, respectively. While the most downregulated genes were related to reproduction (embryogenesis/oogenesis), the repertoire of upregulated genes was overrepresented by genes related to non-self recognition, ubiquitin-protease system, cytoskeletal traffic, cellular proliferation, development and movement, and snRNA processing. Overall, the virus, Musca domestica salivary gland hytrosavirus (MdSGHV), induced the upregulation of various components of the siRNA, innate antimicrobial immune, and autophagy pathways. We show that MdSGHV undergo limited morphogenesis in the corpora allata/corpora cardiaca (CA/CC) complex of $M$. domestica. MdSGHV replication in CA/CC potentially explains the significant reduction of hemolymph sesquiterpenoids levels, the refusal to mate, and the complete shutdown of egg development in viremic females. Notably, hormonal rescue of vitellogenesis did not result in egg production. The mechanism underlying MdSGHV-induced sterility has yet to be resolved.

Keywords: Hytrosaviridae, hypertrophy, sesquiterpenoids, vitellogenesis, corpora allata/corpora cardiaca complex, immunity pathways 


\section{INTRODUCTION}

The family Hytrosaviridae includes a small group of enveloped, rod-shaped dsDNA viruses that infect adult dipterans (AbdAlla et al., 2009). Hytrosaviruses (SGHVs) are defined by their ability to infect and replicate in the salivary glands (SGs) of their hosts and to induce enlarged, swollen glands within which viral progenies are produced and released into the SG lumen. To date, the adult stage of the blood-feeding tsetse fly (Glossina spp.), the filth-feeding housefly (Musca domestica), and a phytophagous syrphid Merodon equestris have been reported to be SGHV hosts (Abd-Alla et al., 2009). However, the lack of overt symptoms, the endemic prevalence of the infection, the lack of susceptible cell lines, and the chronic nature of SGHV infections have hindered the identification of additional SGHV-fly associations (Kariithi et al., 2013). Within the Hytrosaviridae family, GpSGHV infecting Glossina pallidipes and MdSGHV infecting Musca domestica have been sequenced and characterized (Lietze et al., 2011a). Despite inducing similar gross symptoms, these two viruses possess distinct molecular and pathological properties.

The larger GpSGHV, persisting asymptomatically in tsetse, will under certain conditions induce a symptomatic infection resulting in overt SG hyperplasia (SGH) symptoms via gland cell proliferation. The GpSGHV, due in part to the adenotrophic viviparity exhibited by tsetse, can be transmitted readily via the milk glands to developing progeny (Boucias et al., 2013b). Two GpSGHV lineages have been sequenced. The Ethiopian isolate (GpSGHV-Eth) is associated with higher SGH prevalence (85\%) than the Ugandan isolate (GpSGHV-Uga; SGH prevalence of 10\%) (Abd-Alla et al., 2008, 2010, 2011, 2016). The observed differential GpSGHV pathologies in different tsetse colonies have been attributed to genetic differences between the two virus isolates. Compared with GpSGHV-Uga, GpSGHV-Eth contains more ORFs, including 24 novel ORFs not found in GpSGHVUga. Further, 21\% of GpSGHV-Eth ORFs harbor numerous mutations, whereas 6,7 , and $13 \%$ of ORFs are deleted, noncanonical, and inserted, respectively (Abd-Alla et al., 2016). At least 60 of the GpSGHV ORFs encode functional proteins, including homologs to well-characterized baculovirus core genes, helicase 2 (DNA repair and recombination) (Wang et al., 2007), LEFs-4, 5, 8, and 9 (transcription), Ac66 (egress of mature virions), and Ac81 (virus-host interactions at late infection stages) (Miele et al., 2011).

The MdSGHV contains a $126 \mathrm{kbp}$ dsDNA genome encoding for 108 ORFs; the virus induces SGH within 3-4 days post challenge (Garcia-Maruniak et al., 2008). Unlike GpSGHV, MdSGHV causes only symptomatic infections in $M$. domestica and induces SGH by cell hypertrophy but not cell proliferation (Lietze et al., 2011a). With this virus, the SGH is due to massive viral DNA replication and morphogenesis in the SG nuclei, resulting in hypertrophied gland tissue (Lietze et al., 2011a). This virus, although non-lytic, is shed continuously into SG lumens and subsequently deposited in the fly's crop. Adult houseflies exist at high population densities, are gregarious, and feed by regurgitating the contents of the crop (containing saliva-stomach contents) onto the food substrate. Throughout their lifespan, viremic flies release infectious viral particles onto potential food substrates; it is estimated that $\sim 10^{6}$ viral genome copies are released within a $2-3 \mathrm{~s}$ feeding event (Lietze et al., 2009). However, the development of the peritrophic membrane in adult flies serves as an effective barrier to viral ingress in the gut, preventing the development of SGHV-induced epizootics (Prompiboon et al., 2010; Boucias et al., 2015). As an alternative to per os acquisition, cuticle wounding has been proposed as an MdSGHV transmission mode (Lietze et al., 2013). As few as 10 100 virus copies delivered into the hemocoel induce the onset of SGH.

Significantly, in houseflies MdSGHV can undergo both DNA replication and transcription in non-SG tissues, but these events do not result in detectable cytopathology (Lietze et al., 2007, 2011b). Using RT-qPCR, Lietze et al. (2007) demonstrated that, whereas MdSGHV transcription significantly occurred in the fat body, tracheal, and brain tissues, negligible transcripts occurred in the midgut, ovaries, and hemolymph. Transcription in the non-SG tissues is believed to negatively impact reproductive fitness of infected females. At the physiological level, MdSGHV infection blocks vitellogenesis, resulting in immediate and permanent female sterilization. The sterility is absolute; to date, none of thousands of SGH-positive females examined have produced eggs. The mechanism(s) underlying the lack of egg production is unknown, but prior qPCR experiments have shown that fat body transcription of selected female-specific yolk proteins is negligible in virus-infected flies (Lietze et al., 2007). In healthy females, the ingestion of a protein meal is believed to trigger a hormonal cascade involving both juvenile hormone $(\mathrm{JH})$ and ecdysone that activates the transcription of yolk proteins in the fat body (Peferoen and de Loof, 1986; Siegenthaler et al., 2010). It should be noted that limited MdSGHV replication has been observed in the corpora cardiaca (CC), the glands responsible for synthesis of sesquiterpenoids. In dipterans, the released JH-III is believed to trigger the follicle epithelium to produce a pulse of ecdysone that activates fat body biosynthesis of egg proteins. Healthy $M$. domestica females, after access to a protein meal, were shown to produce increased levels of hemolymph-borne methyl farnesoate (MF), the unepoxidated form of JH-III (JH-IIIB), a hormone also synthesized in the corpora allata (CA) (Teal et al., 2014). Therefore, MdSGHV infection of the CA-CC complex potentially disrupts the hormonal cascade required for activation of fat body transcription of female-specific proteins. In addition to blocking egg production, viral infection also alters reproductive behavior, most notably, the refusal of infected females to copulate with either healthy or viremic males (Lietze et al., 2007). We speculate that viral transcription in the neural system may suppress vitellogenesis by modulating neuroendrocrine secretion or through direct regulation of fat body biosynthesis.

Recent availability of the M. domestica genome (Scott et al., 2014) provides a framework to undertake a global examination of the key genes/pathways being regulated by MdSGHV infection. In this study, RNA-Seq data from triplicate bar-coded libraries were generated on the transcriptomes from 
healthy and virus-infected ( $48 \mathrm{~h}$ post infection) flies. These data were annotated against the MdSGHV, M. domestica and Drosophila databases to assess the genes and gene pathways regulated by the SGHV infection. Comparative analysis of the RNA-Seq reads from control and viremic flies demonstrated that MdSGHV infection upregulates components of the innate defense system while suppressing multiple genes involved in oogenesis/egg biosynthesis. Also performed were a series of functional assays targeted at the activities of the antimicrobial peptides (AMPs) and the potential disruption of the hormonal cascade involved in oogenesis in MdSGHVinfected females.

\section{MATERIALS AND METHODS}

\section{Virus Challenge of Houseflies}

Housefly pupae, obtained from colonies maintained at the USDA Center for Medical, Agricultural and Veterinary Entomology (CMAVE, Gainesville, FL), were placed in rearing cages, provided with deionized water, and reared at $26^{\circ} \mathrm{C}$, with a photoperiod of $12 \mathrm{~h}$ light and $12 \mathrm{~h}$ darkness, and $40 \%$ relative humidity until adult emergence. Experiments were conducted with MdSGHV03, the Florida type MdSGHV strain collected in 2005 and subsequently sequenced (Garcia-Maruniak et al., 2008; Prompiboon et al., 2010). Cohorts of synchronously infected houseflies were produced by injecting newly emerged females with filter-sterilized viremic SG homogenates, a treatment that guarantees symptomatic infection in $100 \%$ of the injected flies (Lietze et al., 2007). Control flies were injected with sterile phosphate-buffered saline (PBS). Both PBS- and virus-injected flies were maintained in separate groups in the above-described conditions and provided with food and water ad libitum until used for sample preparation.

\section{RNA Extraction and Quantitation}

At $48 \mathrm{~h}$ post challenge, PBS- and virus-injected females were placed individually in tubes containing aliquots of $1 \mathrm{ml}$ of TriReagent (Sigma-Aldrich, St. Louis, MO). It should be noted that it is at $48 \mathrm{~h}$-pi that injected flies release numerous infectious viral particles, an indication of active virus replication (Lietze et al., 2009). Each sample was homogenized by adding $\sim 20$ zirconium beads (BioSpec Products, Bartlesville, OK) followed by $30 \mathrm{~s}$ of vigorous shaking in a bead-homogenizer (FastPrep ${ }^{\circledR}$ Instrument, Qbiogene, Carlsbad, CA). Total RNA was extracted according to the Tri-Reagent protocol. Ethanol-precipitated RNA pellets were suspended in $100 \mu \mathrm{l}$ DEPC-treated water and treated with RNase-free DNase (Qiagen, Valencia, CA). The isopropanolprecipitated total RNA was re-extracted using SV Total RNA Isolation Kit (Promega, Fitchburg, WI). RNA quantity and quality were assessed using a NanoDrop 2000 spectrophotometer (Thermo Scientific, Wilmington, DE) and the Agilent 2100 Bioanalyzer (Agilent Technologies, Inc., Santa Clara, CA). The absence of contaminating DNA in RNA samples was verified using conventional PCR amplification primers targeting the $28 \mathrm{~S}$ rRNA gene (GenBank accession number DQ656974) (Salem et al., 2009).

\section{RNA-Seq Library Construction and Data Analysis}

Preparation and sequencing of RNA libraries were performed by ICBR/UF (Gainesville, FL) according to the manufacturer's instructions (Illumina, Inc., San Diego, CA) using the NextSeq500 platform. Briefly, the mRNA was enriched from $1 \mu \mathrm{g}$ of total RNA per sample using oligo-dT attached to magnetic beads and then subjected to thermal fragmentation using the elute, prime, and fragmentation mix from the Illumina TruSeq $^{\text {TM }}$ v2 RNA sample preparation kit. RNA fragments were then converted to double-stranded (ds)-cDNA using reverse transcriptase and random primers provided in the TruSeq RNA sample preparation kit. The ds-cDNA fragments were end-repaired by enzymatic polishing with T4 DNA polymerase and E. coli DNA polymerase I Klenow fragment. A single non-templated dA-tail was added to the $3^{\prime}$-end of the repaired fragments and then ligated to NEB adaptors (NEBNext ${ }^{\circledR}$ Ultra RNA library preparation kit). The required fragments were purified by AMPure beads (Agencourt; PN A63881) and enriched by PCR amplification. The amplified libraries were purified and quantified using the Agilent DNA high-sensitivity kit on an Agilent 2100 Bioanalyzer (Agilent Technologies, Inc.) and qPCR. Based on the calculated values, the libraries were pooled in equimolar ratios into one pool and sequenced for 2 $\times 150$ bp reads on the Illumina NextSeq 500 platform. Image analysis and base calling were performed using the Illumina Pipeline, where sequence tags were obtained after purity filtering.

Reads acquired from Illumina were cleaned up with the Cutadapt program to trim off sequencing adaptors, low-quality bases with a quality phred-like score $<20$, and short reads $(<40$ bases) (Martin, 2011). The genes or transcripts of $M$. domestica (38,323 sequences) from NCBI were used as reference sequences for RNA-Seq analysis. The cleaned reads of each sample were mapped independently against the reference sequences using the mapper of Bowtie 2 with a maximum of three mismatches for each read (Langmead and Salzberg, 2012). The mapping results were processed with Samtools and scripts developed inhouse at ICBR to remove potential PCR duplicates and to select unique mapping reads for gene expression estimation. Digital gene expression was determined by counting the numbers of mapped reads for each individual gene counted, using the scripts developed in-house at ICBR and analyzed by the DEB application (Yao and $\mathrm{Yu}, 2011$ ).

\section{Assignments of Gene Ontology (GO) Terms and Pathway Analyses}

All genes with padj $\leq 0.2$ were selected for the GO analysis. In these selected genes or transcripts, detailed information on the genes was retrieved from the reference databases of $M$. domestica and/or Drosophila. In the GO analysis, the levels of upregulation (fold-change $>0$ ) and downregulation (fold-change $<0$ ) were based on the log transformed-fold-change of the RNA-seq results. For pathway analysis annotated unigenes selected at high stringency (padj $\leq 0.01)$ were divided into two pools: the downregulated pool $(n=446)$ and an upregulated gene pool $(n=$ 527 ), having $\log 2$ fold values of $\leq 2.0$ and $\geq 2.0$, respectively. The 
amino acid sequences of the $M$. domestica unigenes were blasted against the non-nr-NCBI by BLASTp $\left(e\right.$-value $\left.10^{-4}\right)$ and the GOs enriched with Fisher's exact test (cut-off FDR of $<0.05$ ) using Blast2GO v4.0 (Conesa et al., 2005). Detailed information of the genes was retrieved from the reference databases of $M$. domestica and/or Drosophila. Pathway analyses were performed using the KEGG Mapper v2.7 (Kanehisa et al., 2004) and Insect Innate Immunity Database (IIID) (Brucker et al., 2012). Analysis of the structural features and functional domains of the MdSGHV ORFs was performed using various databases, including PREDTMBB (Bagos et al., 2004), Pfam v30.0 (Finn et al., 2008), and the NCBI's conserved domain database (CDD) v3.15 (MarchlerBauer et al., 2015).

\section{RT-qPCR Validation}

RNA samples from PBS- and virus-injected female flies were subjected to a one-step RT-qPCR to quantitate the relative transcript abundance of 23 genes selected from the RNA-Seq data. Primers were designed using Primer3 Plus (Untergasser et al., 2007) to amplify 125-200 bp from M. domestica genes predicted from the RNA-Seq reads (Table S1). In addition to host gene targets, primers designed to amplify ORFs 1, 10, and 108 (Lietze et al., 2011b) were used to confirm the infection status of the virus-injected samples. The $M$. domestica $28 S r R N A$ gene served as a reference gene and an internal positive control. Using the iTaq ${ }^{\mathrm{TM}}$ Universal SYBR ${ }^{\circledR}$ Green One-Step kit (Bio-Rad, CA), each $20-\mu 1$ reaction contained 50 ng of DNase-treated total RNA, 5 pmol of each gene-specific forward and reverse primer, and the reaction mix with iScript ${ }^{\circledR}$ reverse transcriptase. The one-step RT-qPCR program was one reverse transcription cycle $\left(50^{\circ} \mathrm{C}\right.$ for $10 \mathrm{~min})$, one initial denaturation cycle $\left(95^{\circ} \mathrm{C}\right.$ for $3 \mathrm{~min}$, ), then 40 cycles of $95^{\circ} \mathrm{C}$ for $10 \mathrm{~s}, 60^{\circ} \mathrm{C}$ for $30 \mathrm{~s}$ and melt curve analysis of $65-95^{\circ} \mathrm{C}$ at $0.5^{\circ} \mathrm{C}$ increments ( $5 \mathrm{~s}$ per step). The melting peaks were inspected to confirm the presence of a single-amplification PCR product for each reaction. The relative quantification of target gene expression in the virus-injected samples was analyzed using the $2^{-\Delta \Delta C T}$ method (Livak and Schmittgen, 2001). The qPCR data were presented as the fold change (FC) in the target gene normalized to the $28 S$ rRNA gene and relative to the PBSinjected control samples. Specifically, the $C_{\mathrm{T}}$ values of the target genes were subtracted by the $C_{\mathrm{T}}$ values of $28 \mathrm{~S}$ rRNA gene. Then, the FC in the target genes, relative to the PBS-injected control, was calculated for each virus-injected sample and then $\log _{2}$-transformed. The $\log _{2}$-transformed FC means and standard errors (SE) were determined from the triplicate samples for each target gene.

\section{Evaluation of Endogenous Levels of Sesquiterpenoids}

A series of bioassays were conducted to compare the endogenous levels of JH-III (methyl (2E,6E)-10,11-epoxy-3,7,11-trimethyl2,6-dodecadienoate), a JH-IIIB analog (methyl (2E,6E)6,7;10,11-bisepoxy-3,7,11-trimethyl-2-dodecenoate), and the JH-III biosynthetic precursor MF (methyl (2E,6E)-3,7,11trimethyldodeca-2,6,10-tri-enoate) in MdSGHV-challenged and control flies. Fifty newly emerged females were injected with either sterile saline (control) or with viremic SG homogenates.
Flies, provisioned with adult food and water, were incubated at $26^{\circ} \mathrm{C}$. At $1,2,3$, and 4 days post injection (d-pi), flies were cold-immobilized and hemolymph collected by cutting a hind leg and withdrawing extruded hemolymph into a pre-chilled $10 \mu \mathrm{l}$ capillary. Collected hemolymph samples were transferred into HPLC-grade methanol. The sesquiterpenes in methanol were extracted with pentane to remove lipids and analyzed using a combination of GC and ionization mass-spectroscopy (Teal et al., 2014). Flies used in these extractions were dissected to assess both their SGH status and ovarian development stage.

\section{Evaluation of Impacts of Exogenous Hormones on MdSGHV-Induced Pathologies}

To evaluate effects of exogenous hormones on ovarian development, females were PBS- and MdSGHV-injected. After $24 \mathrm{~h}$ post injection (h-pi), the flies were cold-immobilized and injected (500 ng per fly) with either ecdysone suspended in $10 \%$ ethanol, commercial JH-III (Sigma Chemical, St. Louis, MO), or methyl farnesoate (Echelon BioSciences, Salt Lake City, UT) dissolved in acetone $(100 \mathrm{mg} / \mathrm{ml})$ and suspended in peanut oil $(1 \mathrm{mg} / \mathrm{ml})$. Controls included groups of flies injected with an acetone-peanut oil mixture. The treated females were placed in holding cages with 20 healthy males and provisioned adult food and water. After $5 \mathrm{~d}$-pi, the females were dissected to determine SGH symptoms and ovarian development stages. Another treatment involved injecting a cocktail containing JH-III, MF, and ecdysone mixed with PBS or with SGHV into newly emerged females. Flies were injected with $1 \mu \mathrm{l}$ containing $500 \mathrm{ng}$ of each hormone suspended in PBS with and without MdSGHV. After $5 \mathrm{~d}$-pi, females were dissected to determine SGH and ovarian development.

Additional groups of females injected initially with either PBS or virus homogenate were incubated for $24 \mathrm{~h}$ at $28^{\circ} \mathrm{C}$ and re-injected with solvent (10\% ethanol), $0.1 \mu \mathrm{g}$ ecdysone, or $1 \mu \mathrm{g}$ ecdysone. Treated flies were maintained in separate cages on their respective diets of either $10 \%$ sucrose in water or water plus adult food (powdered milk + sucrose). After a 2day incubation period, three females per treatment were collected individually, weighed, and dissected in sterile saline to record SGH and ovarian development. At 4 d-pi, total RNA and cDNA were prepared as described in sections 2.2 and as described in Lietze et al. (2007), followed by RT-qPCR using the iQTM SYBR $^{\circledR}$ Green Supermix (Bio-Rad, Hercules, CA). RT-qPCR was performed using an optimized protocol for three designed primer sets specific for $M$. domestica hexamerin (Hex2), yolk protein (Yo2), and the 28S rRNA housekeeping genes (Lietze et al., 2007).

\section{Impact of MdSGHV Infection on the Innate Defense Response}

To determine if the increased transcript levels impacted the hemolymph AMP titers and/or microbiome associated with viremic flies, cohorts of newly eclosed females were injected with PBS-homogenates of either healthy or hypertrophied SGs. Additional controls included non-injected females and females injected with E. coli D31 ( $10^{6}$ cells per fly). At $1-10$ d-pi, 
groups of five flies were homogenized in liquid $\mathrm{N}_{2}$, resuspended in $400 \mu \mathrm{l}$ of $4 \%$ acetic acid, vortexed, heated $\left(95^{\circ} \mathrm{C} ; 5 \mathrm{~min}\right)$, and centrifuged $\left(12,000 \mathrm{rpm} ; 15 \mathrm{~min} ; 4^{\circ} \mathrm{C}\right)$. Supernatants were lyophilized and stored $\left(-70^{\circ} \mathrm{C}\right)$ until assayed. The relative AMP activity was assessed using the inhibition zone assay (Hultmark, 1998). In brief, freeze-dried preparations were solubilized in 30 $\mu \mathrm{l}$ sterile PBS and applied to wells $(\sim 2.0 \mu \mathrm{l}$ per well $)$ of $\mathrm{LB}$ agar plates pre-inoculated with early exponential growth phase Serratia marcescens or E. coli D31. In addition to gram-negative bacteria, plates seeded with a preparation of Micrococcus luteus (1 $\mathrm{mg} / \mathrm{ml}$, Sigma) or with live Saccharomyces cerevisiae were used as substrates to measure relative lysozyme and anti-yeast activities. After $24 \mathrm{~h}$ at $28^{\circ} \mathrm{C}$, all the above-mentioned plates were examined to estimate the area of inhibition zones. The potential impact of the upregulation of the innate defenses by MdSGHV infection on the housefly microbiome was estimated by calculating the total cultivable colony forming units (CFUs). Three cohorts of newly eclosed female and male adult houseflies were PBS- or MdSGHV-injected and maintained on adult food and water. After 48, 72, and 96 h-pi, flies were individually homogenized for $10 \mathrm{~s}$ in $1 \mathrm{ml}$ water using Tissuemiser ${ }^{\mathrm{TM}}$ (Fisher Scientific). Resultant homogenates were serially diluted in water and decimal dilutions subsequently spotted $(2 \mu \mathrm{l}$ per spot) onto nutrient agar plates. After $24 \mathrm{~h}$ at $28^{\circ} \mathrm{C}$, plates were examined to estimate the total CFUs contained in individual flies.

\section{RESULTS}

\section{Viral (MdSGHV) RNA-Seq Reads}

Mapping the RNA-Seq data onto the MdSGHV genome (GenBank accession number NC_010671) demonstrated that the three PBS-injected (healthy) RNA pools had a total of only 1,528 reads (out of $>2.8 \times 10^{7}$ unique mapped reads obtained) that mapped onto the MdSGHV ORFs. In general, the reads detected in the PBS-injected samples mapped mainly onto the highly expressed virus ORFs 37 (a small nuclear RNA-activating complex subunit 2 (SnAPC-2)-like protein), 40, 48 (a N. meningitidis TspB virulence factor-like protein), 86 (matrix protein), MdSGHV093, and MdSGHV096 (Table S2). Alternatively, at $48 \mathrm{~h}$-pi, the RNA-Seq data from the three virusinfected RNA pools contained $4.5 \times 10^{6}, 4.2 \times 10^{6}$, and 2.0 $\times 10^{6}$ reads, respectively, that could be mapped onto all of the 108 MdSGHV ORFs (Garcia-Maruniak et al., 2009) (Figure 1). Overall, the number of reads was not correlated $\left(R^{2}=0.056\right)$ to size of the ORFs; however, the six ORFs having the smallest number of reads were $>300$ bp in length (Figure S1). Based on the read frequencies, viral transcription appeared to be regulated; 15 ORFs (highly abundant) had more than $5.0 \times 10^{4}$ reads per library, 39 ORFs (abundant) had between 1.0 and $5.0 \times 10^{4}$ reads per library, 37 ORFs (moderate) had between 10.0 and $2.0 \times 10^{3}$ reads per library, and 11 ORFs (low) had between 20.0 and $2.0 \times$ $10^{2}$ reads per library (Figure 2 ).

\section{Host (M. domestica) RNA-Seq Reads}

The three libraries generated for the individual PBS-control and MdSGHV-infected females produced similar numbers of raw forward and reverse reads (Table 1) that mapped onto the $M$. domestica genome (GenBank accession number AQPM00000000.1). The reduced number of unique mapped reads detected in virus-infected libraries is likely due to the presence of the millions of reads matching the viral ORFs that were absent in healthy $M$. domestica (see prior section). After filtering out low-quality mappings, 20,197 genes were selected for further analysis. In the RNA-Seq analyses, genes with low ( $<10$ reads) or no reads in any of the libraries were discarded, leaving $\sim 17,000$ putative gene targets. Comparative analysis between the mapped reads (17,000 putative genes) of the healthy (control) and infected libraries identified $\sim 2,300$ (14\%) and $\sim 5,500$ (32\%) having padj $\leq 0.01$ and $<0.2$, respectively, that were modulated to various levels by MdSGHV infection. Of the $\sim 5,500$ differentially expressed genes (padj $<0.2$ ), similar numbers were placed into the positive $\log 2 \mathrm{FC}(2,7)$ and the negative $\log 2 \mathrm{FC}(-2,7)$ pools. Within these unigene gene pools (padj $<0.2$ ), 858 and 559 unigenes had $\log 2 \mathrm{FC}$ of $\geq 2.0$ or $\leq-2.0$, respectively.

\section{Validation of RNA-Seq Data by RT-qPCR}

The RT-qPCR assays targeting MdSGHV ORFs 1 (DNA pol), 10 (mitochondrial carrier), and 108 (Ac81) demonstrated that the control libraries lacked the virus $\left(C_{\mathrm{T}}\right.$ values $\left.>35\right)$. Further, the copy numbers of MdSGHV010, a gene having an abundant level of mapped reads in the RNA-Seq analysis, was estimated to be seven-fold greater than those generated for the moderately expressed MdSGHV001 (Figure 2). The qPCR assays, conducted on 22 host genes regulated to varying degrees by MdSGHV infection, produced data that supported the RNA-Seq findings (Figure 3). In certain cases, there were disparities in the folddifference between these data sets. For example, the pronounced increase (128- to 512-fold) in the transcript levels for cationic peptides (diptericin-D, sarcotoxin II-1, and attacin-A) observed in the RNA-Seq data were not observed in the qPCR assays; in these reactions, only 4 - to 8 -fold increases were recorded for these gene targets. On the other hand, RT-qPCR data recorded lower transcript levels in eight of the eleven genes identified by RNASeq analysis to be suppressed by MdSGHV infection (Figure 3). It should be noted that the variation between RNA-Seq and RT-qPCR data may be due to biological replication; the qPCR analyses were conducted on RNA preparations that were not the same as the RNA preparations used to prepare the libraries for the RNA-Seq analyses.

\section{Gene Ontology (GO) Classification}

Many of the differentially expressed host unigenes (padj $<0.2$ ) could be annotated and assigned via GO annotation into the three major categories: the biological process (BP; 2,271 unigenes), the cellular component (CP; 1,980 unigenes), and the molecular function (MF; 2,462 unigenes) ontologies (Figure 4). Within the major $\mathrm{GO}$ categories, genes having negative $\log 2 \mathrm{FC}$ values (downregulated category) outnumbered by $\sim 1.5$ - to 2 -fold those genes having positive $\log 2 \mathrm{FC}$ values (upregulated category). It should be noted that increased levels within the downregulated category in the GO analysis is somewhat biased; more unigenes 


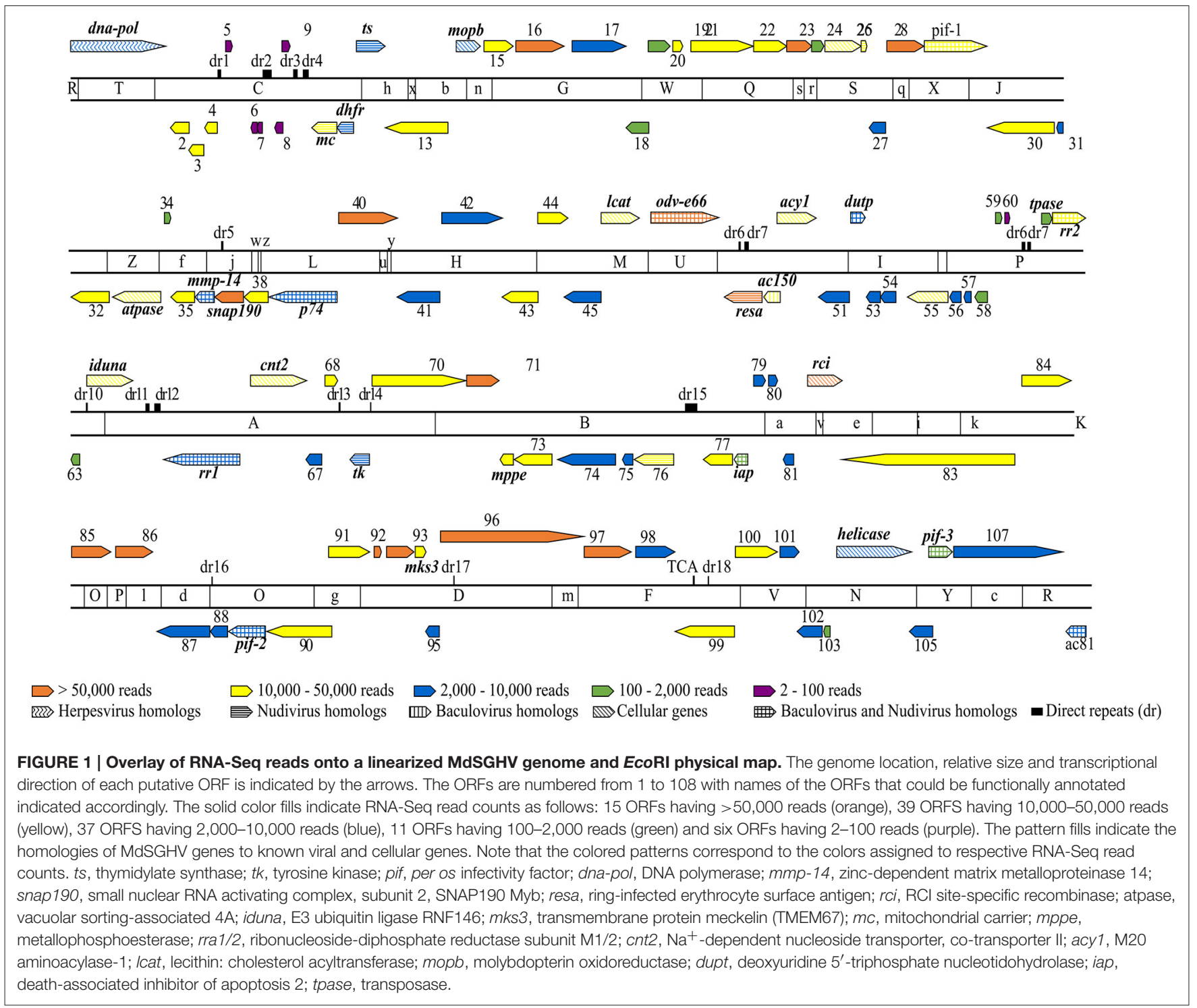

$(1,664)$ that could not be assigned a GO category $(n=2,713)$ had a positive $\log 2 \mathrm{FC}$ value.

Viral infection at $48 \mathrm{~h}$-pi altered transcription profiles in many of the GO subcategories within the $\mathrm{BP}, \mathrm{CP}$, and MF ontology (Figure 4). Within most of the BP subcategories, there were 1.3- to 2.6-fold more genes having downregulated transcript levels compared to the controls. The three subcategories of reproduction, reproductive process, and negative regulation of biological process had more than a 3 -fold increase in the number of downregulated transcripts. There were exceptions to the general reduction in transcript levels; for example, both immune and biological adhesion subcategories within the BP ontology contained more genes having a positive than a negative $\log 2 \mathrm{FC}$. Like the BP, within the CP, the majority of subcategories had reduced transcript levels; marked downregulation was noted with transcripts in the membrane-enclosed lumen extracellular region category. This subcategory included genes associated with organelles enclosed by double membranes (endoplasmic reticulum, nucleus). However, the extracellular space and matrix and cell junction subcategories contained more genes with upregulated than downregulated transcript levels. Within the $\mathrm{MF}$, the overall degree of the gene downregulation induced by MdSGHV infection was somewhat less than in the BP and CP categories. In the MF category, the transporter activity, enzyme regulatory, electron carrier, and receptor subcategories had more unigenes having a positive than a negative $\log 2 \mathrm{FC}$.

\section{Differentially Expressed Host Genes}

Two sets of unigenes (padj $\leq 0.01$ ) exhibiting the greatest $\log 2 \mathrm{FC}$ reductions or increases in transcript levels were annotated against a combination of $M$. domestica and Drosophila databases. Annotated genes having the lowest $\log 2 \mathrm{FC}$ values $\left(2^{-10}\right.$ to $2^{-4}$ ) were dominated by the genes encoding for femalespecific proteins related to reproduction, egg production, and 

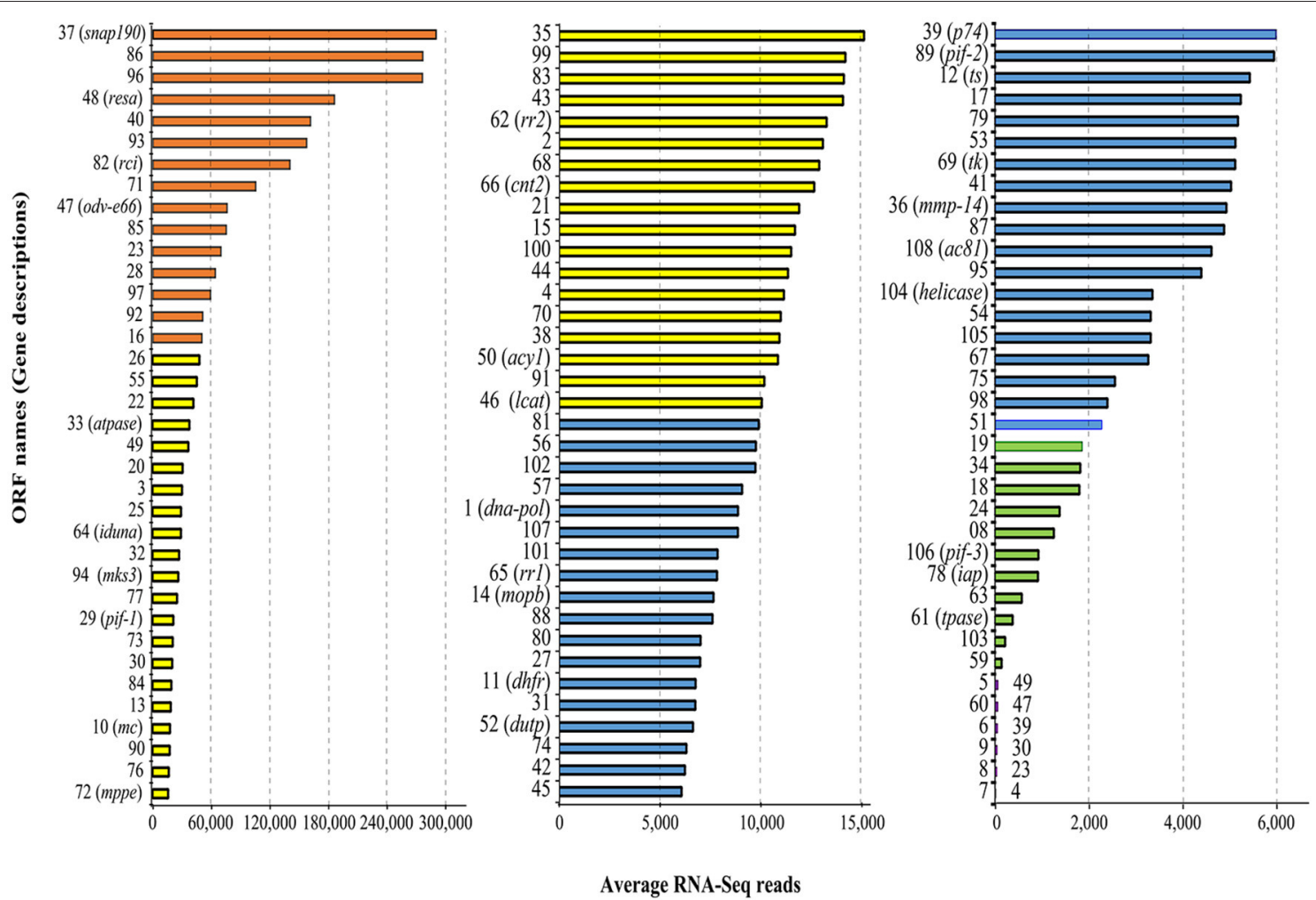

FIGURE 2 | Average number of RNA-Seq reads in viremic libraries associated with MdSGHV ORFs. Note the change of scale on $\mathrm{x}$-axis in the three figure panels. The total numbers of the ORFs and the colors in this figure correspond to Figure 1. ts, thymidylate synthase; tk, tyrosine kinase; pif, per os infectivity factor; dna-pol, DNA polymerase; mmp-14, zinc-dependent matrix metalloproteinase 14; snap190, small nuclear RNA activating complex, subunit 2, SNAP190 Myb; resa, ring-infected erythrocyte surface antigen; rci, RCl site-specific recombinase; atpase, vacuolar sorting-associated 4A; iduna, E3 ubiquitin ligase RNF146; mks3, Transmembrane protein Meckelin (TMEM67); mc, mitochondrial carrier; mppe, metallophosphoesterase; rra1/2, ribonucleoside-diphosphate reductase subunit M1/2; cnt2, $\mathrm{Na}^{+}$-dependent nucleoside transporter, co-transporter II; acy1, M20 Aminoacylase-1; Icat, lecithin: cholesterol acyltransferase; mopb, molybdopterin oxidoreductase; dupt, deoxyuridine $5^{\prime}$-triphosphate nucleotidohydrolase; iap, death-associated inhibitor of apoptosis 2; tpase, transposase.

embryogenesis (yolk proteins, vitellogenins, nudel, hexamerin, meiosis arrest female 1 , protein takeout, bicaudal $\mathrm{C}$, chorion peroxidase, pendulin) (Table S3). In addition, other annotated genes expressing high $\log 2 \mathrm{FC}$ reductions included transcripts encoding for proteins involved in non-self-recognition (lectin subunit alpha); SG associated endonucleases (Tsal1 precursor, Tsal2-A); ubiquitin-proteasome (E3 ubiquitin-ligase UBR1, F-box only 33, MKRN2 opposite strand protein); intracellular movement (kinesin-like protein); lipid metabolism (phospholipid-transporting ATPase IF, acyl-COA binding-like, mitochondrial D-beta-hydroxybutyrate); starch metabolism (amylases); cell proliferation, differentiation, and movement (dual specificity tyrosine-phosphorylation-regulated kinase 3 homolog, epithelial discoidin domain containing receptor-1 serine/threonine aurora 2); and snRNA processing (integrator complex-10).

The 50 genes having the greatest $\log 2 \mathrm{FC}$ increases $\left(2^{4}\right.$ to $2^{9}$ ) included 45 annotated genes, nearly half of which were components or products of humoral and cellular innate defense responses (Table S4). These included various cationic peptides (attacins, sarcotoxins/cecropins, and diptericins), apoptosisrelated genes (peroxiredoxin 3), transcriptional factors (relish), coagulation components (proclotting enzyme), and endocytosis (low density liporeceptor adapter 1, acyl-COA-thioesterase 1 , and calpain-C). Other genes with increased transcript levels encoded for extracellular matrix-associated proteins (laminin subunit gamma-1, heparan sulfate 2-O-sulfotransferase pipe, prolyl 4-hydroxylase- $\alpha-2$, fibronectin type III domaincontaining 5 - isoform, dachsous), glycosyl hydrolases (maltases, $\alpha-N$-acetylgalactosaminidase), stress-associated (Hsp70), lipid metabolism (elongation of very long-chain fatty acid protein, low-density lipoprotein receptor adapter protein 1-A), transposable element (blastopia polyprotein), cuticle synthesis (cuticle 8, pupal cuticle G1A, laccase, larval cuticle-8), and RNA-binding proteins (couch potato).

\section{Pathway Analysis}

In total, 124 of the 973 annotated unigenes (padj $\leq 0.01$, $\log 2 \mathrm{FC} \leq-2.0$ or $\geq 2.0$ ) could be placed into one or more of 101 KEGG pathways. The low number of genes that could be assigned accurately to specific target pathways is probably due to the nature of multifunctional proteins, which perform several functions simultaneously or not. Only 42 of the 446 downregulated genes could be placed into 
TABLE 1 | Summary statistics of the NextSeq FC bidirectional reads (R1 and R2) generated on the six libraries prepared from total RNA extracted from the PBS-injected (Control) and virus-injected (MdSGHV) Musca domestica female flies.

\begin{tabular}{|c|c|c|c|c|c|c|c|}
\hline RNA sample & \multicolumn{2}{|c|}{ Raw data } & \multicolumn{2}{|c|}{ Cleanup data } & \multicolumn{2}{|c|}{ mRNA mapped reads ${ }^{a}$} & $\frac{\text { mRNA unique mapped reads }^{\mathbf{a}}}{\mathrm{R} 1+\mathbf{R} 2}$ \\
\hline Control 4 & $4.83 \mathrm{E}+07$ & $4.83 \mathrm{E}+07$ & $4.83 \mathrm{E}+07$ & $4.83 \mathrm{E}+07$ & $3.91 \mathrm{E}+07$ & $3.70 \mathrm{E}+07$ & $9.96 \mathrm{E}+06$ \\
\hline Control 5 & $3.86 \mathrm{E}+07$ & $3.86 \mathrm{E}+07$ & $3.86 \mathrm{E}+07$ & $3.86 \mathrm{E}+07$ & $2.93 \mathrm{E}+07$ & $2.77 \mathrm{E}+07$ & $7.45 \mathrm{E}+06$ \\
\hline MdSGHV 2 & $5.26 \mathrm{E}+07$ & $5.26 \mathrm{E}+07$ & $5.26 \mathrm{E}+07$ & $5.26 \mathrm{E}+07$ & $2.84 \mathrm{E}+07$ & $2.71 \mathrm{E}+07$ & $7.69 \mathrm{E}+06$ \\
\hline
\end{tabular}

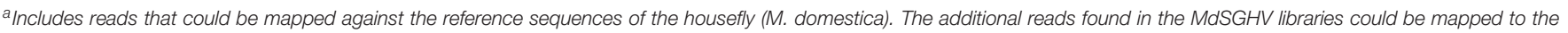
ORFs encoded by the MdSGHV genome.

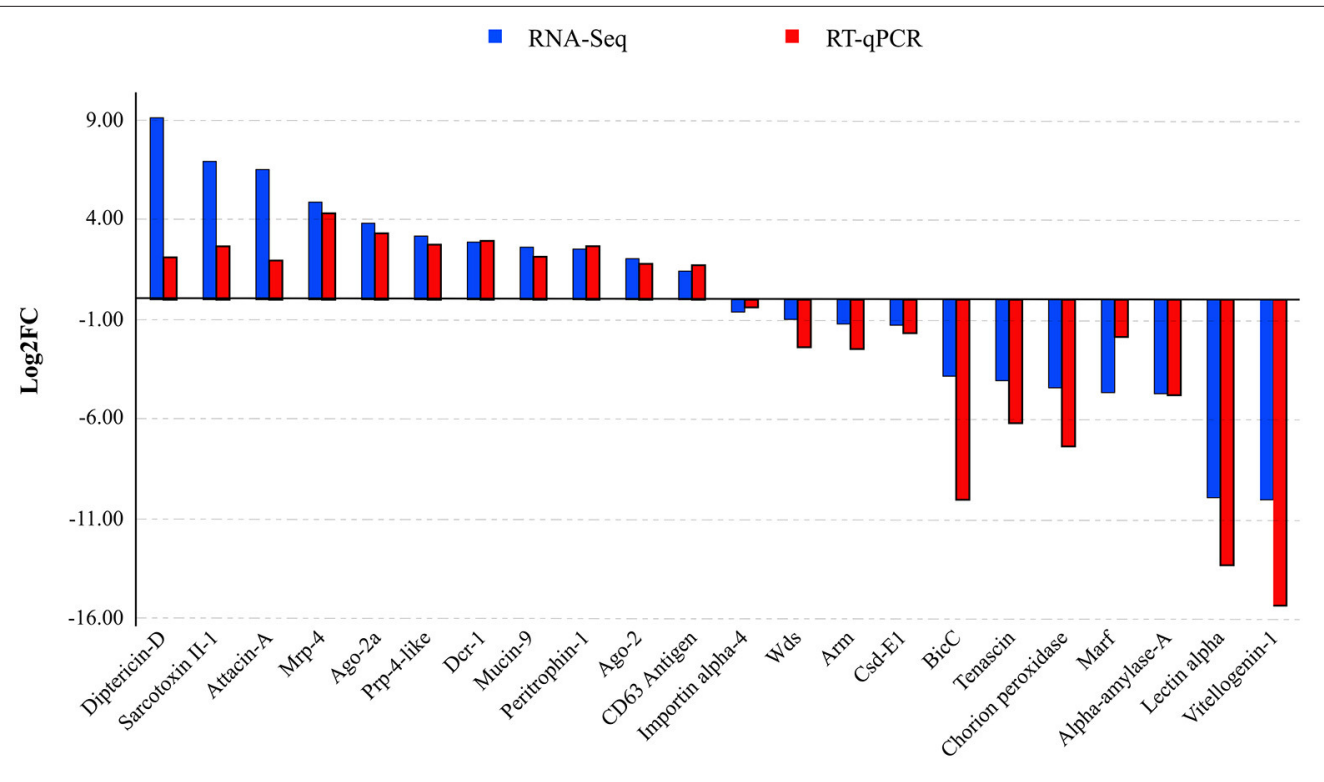

Gene descriptions

FIGURE 3 | MdSGHV-induced modulation of expression of 22 host genes. Except few disparities such as pronounced log2FC of diptericin-D, sarcotoxin II-1, and attacin-A, the RT-qPCR quantification of the host genes correlated with the RNA-Seq data. Mrp-4, Multi-drug resistance-associated protein-4; Prp-4-like, Proline-rich protein 4-like; Wds, Protein will die slowly; Csd-E1, Cold shock domain-containing protein-E1; BicC, Protein bicaudal C; Marf, Meiosis arrest female protein; Argo, Argonaute, Dcr, Endoribonuclease dicer.

one or more of 56 KEGG pathways (Table S5); 25 pathways contained only downregulated $(\leq-2.0)$ genes. Within this pool of 25 pathways, MdSGHV infection lowered the transcript levels of various genes involved in nucleic acid replication and transport ( $\alpha$-primase complex, MCM complex, helicase, and nucleoporins $\mathrm{Ndc1}$ and Nup153) and in DNA repair pathways (endonucleases, DNA excision repair protein, DNA polymerases, and exonuclease). Additionally, components of the Wnt signaling pathways, including the F-box/WD repeat-containing protein 11, serine/threonine-protein (nemo-like) kinase NLK, vanglike protein 2-B, and 1-phosphatidylinositol 4,5-bisphosphate phosphodiesterase, had reduced transcript levels in infected females. These transcripts were distributed within branches of the canonical pathway, the planar cell polarity (PCP) pathway, and the Wnt/Ca ${ }^{2+}$-pathway.

In total, 82 of the 527 upregulated genes could be placed into one or more of 70 KEGG pathways (Table S6); 39 pathways contained only upregulated transcripts. Within the 39 upregulated pathways pool, increased transcript levels of enzymes (UDP-glucuronosyltransferases, uridine phosphorylase, glutathione S-transferases, dihydropyrimidine dehydrogenases, and xanthine dehydrogenase) involved in xenobiotic (drug) biodegradation and metabolism were induced by MdSGHV infection. Viremic flies had increased transcript titers of genes involved in carbohydrate metabolism, including those associated with chitin, glycan, amino-sugar, and pentose metabolism. 
Biological Process (BP)

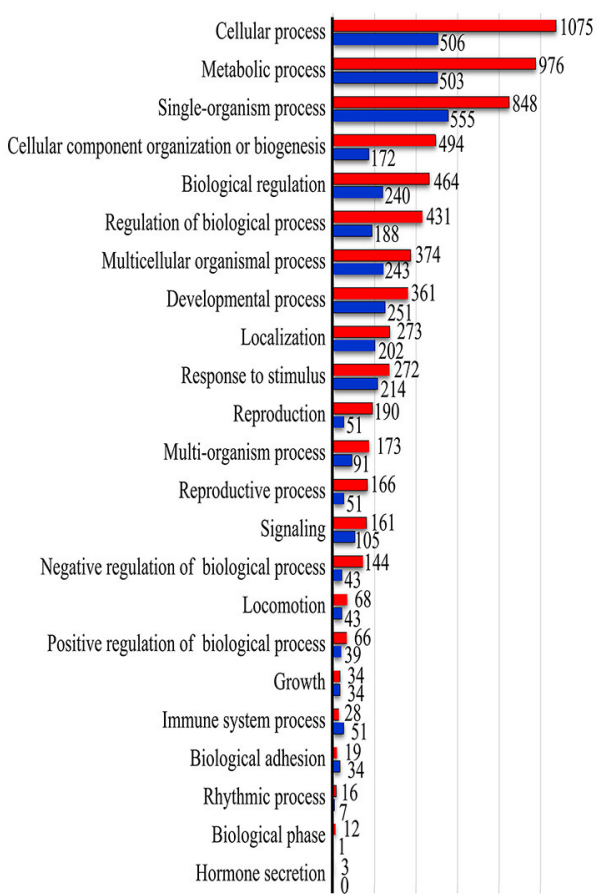

$0 \quad 2004006008001,000$

- Downregulated genes

1. Upregulated genes
Cellular Component (CC)

Molecular Function (MF)

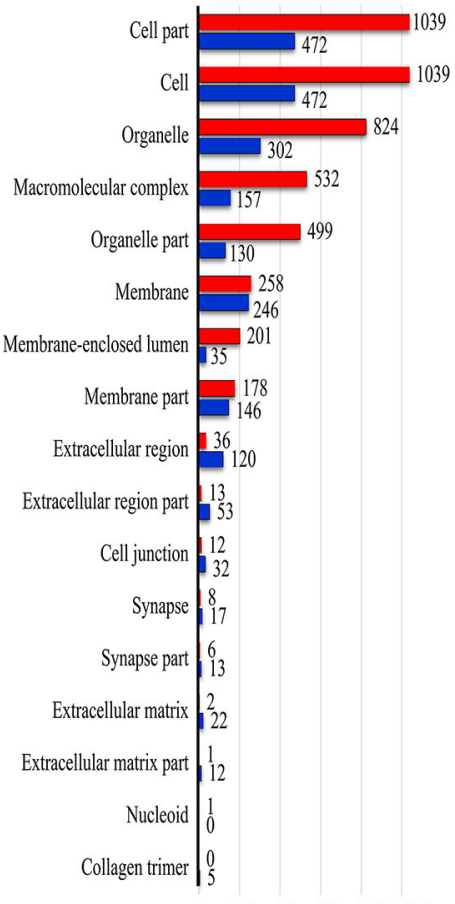

$0 \quad 2004006008001,000$

Number of genes

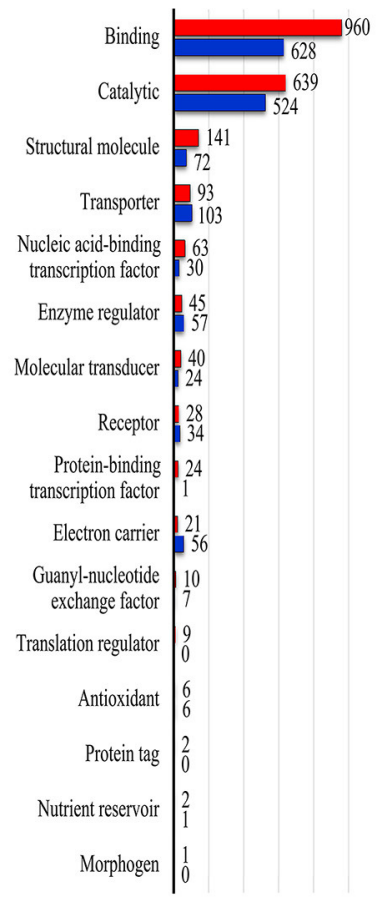

02004006008001,000

Number of genes

FIGURE 4 | GO terms associated with RNA-Seq reads from viremic and healthy housefly libraries. The GO terms were mapped to individual genes that had a padj $<=0.2$. The levels of upregulation (red, fold change $>2.0$ ) and downregulation (blue, fold change $<-2.0$ ) were based on the log transformed-fold-change of the RNA-Seq reads.

The 31 pathways containing a mix of upregulated and downregulated transcripts demonstrated that the differentially expressed transcripts often targeted different sections/branches of a pathway (see Tables S5, S6). For example, in the pyrimidine and purine pathways, the downregulated transcripts encoded for enzymes involved in nucleic acid strand synthesis, whereas the upregulated transcripts encoded enzymes targeting nucleotide catalysis/turnover ( $5^{\prime}$ nucleotidase, adenosine deaminase, adenylate cyclase). The insect hormone pathway contained reduced transcript levels of cytochrome P450 307al (spook) and cytochrome P450 18al involved in ecdysone biosynthesis and 20hydroxyecdysone (20-E) inactivation, respectively, and increased transcript levels of enzymes involved in MF and JH-III biosynthesis. In the extracellular matrix (ECM) pathway, the collagen transcript level was increased, whereas the laminin transcript level was reduced. Certain pathways contained a combination of multifunctional genes and pathway-specific genes identified either in the upregulated or downregulated transcript pools. For example, the hippo signaling pathway contained several multifunctional upregulated genes (protocadherin and F-actin) and various downregulated genes that include pathway-specific serine/threonine-protein kinase Warts.

Due in part to the inability to map the annotated innate defense genes into KEGG pathways, a Top BLASTp search on the IIID database was conducted on the 973 annotated unigenes (padj $\leq 0.01, \log 2 \mathrm{FC} \leq-2.0$ or $\geq 2.0$ ). Forty-two out of the 527 upregulated genes were identified as components/products of both the humoral and cellular immune pathways (Table 2). These included genes encoding for the cationic peptides (AMPs), components of the Toll and Imd pathways, nonself-recognition proteins, phenoloxidase cascade, antiviral defense, and stressrelated proteins. Only 12 of the 446 downregulated genes could be associated with genes in the IIID (Table 3). In most cases, these associations reflected the dual role that components of some of these pathways may play in insect immunity, reproduction, and development. For example, the female-specific hexamerin and vitellogenin receptor are associated with vitellogenesis and chorion peroxidase, nudel and nanos are involved in embryogenesis. In this list, there were genes associated with the negative regulation of Jak/STAT, including E3 SUMO-protein ligase PIAS2 that, when activated by phosphorylation (aurora kinase), inhibits STAT signaling. 


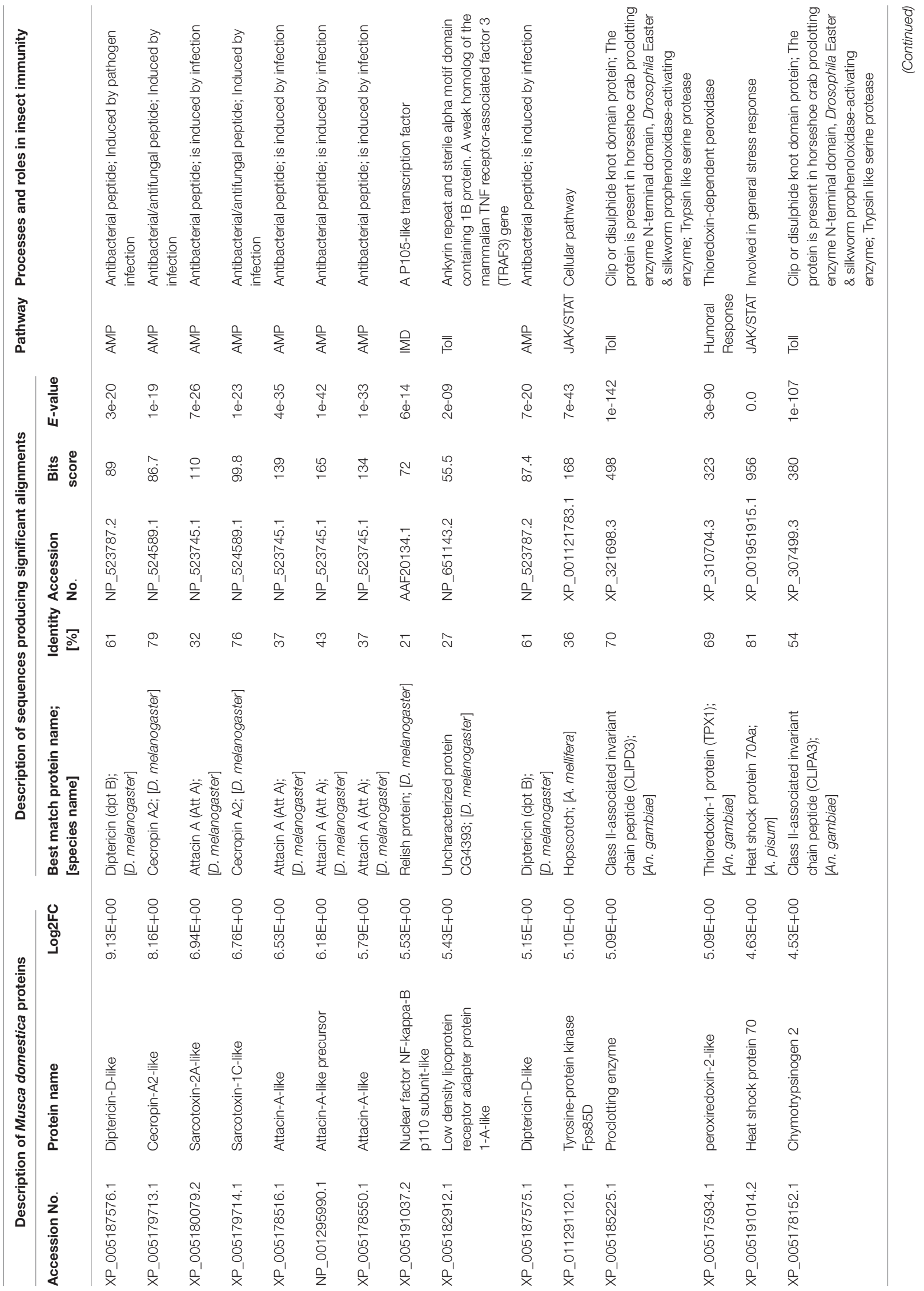




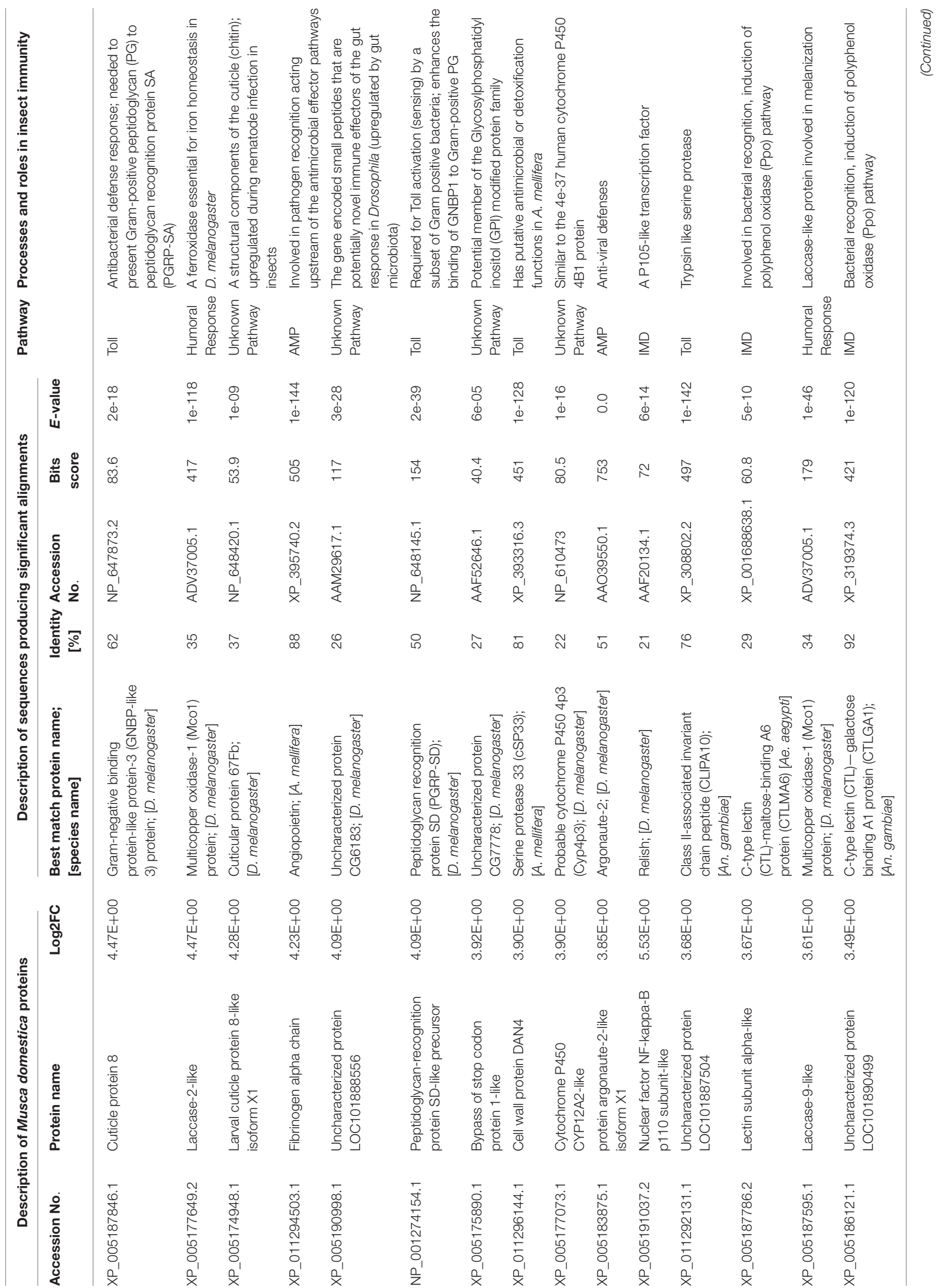




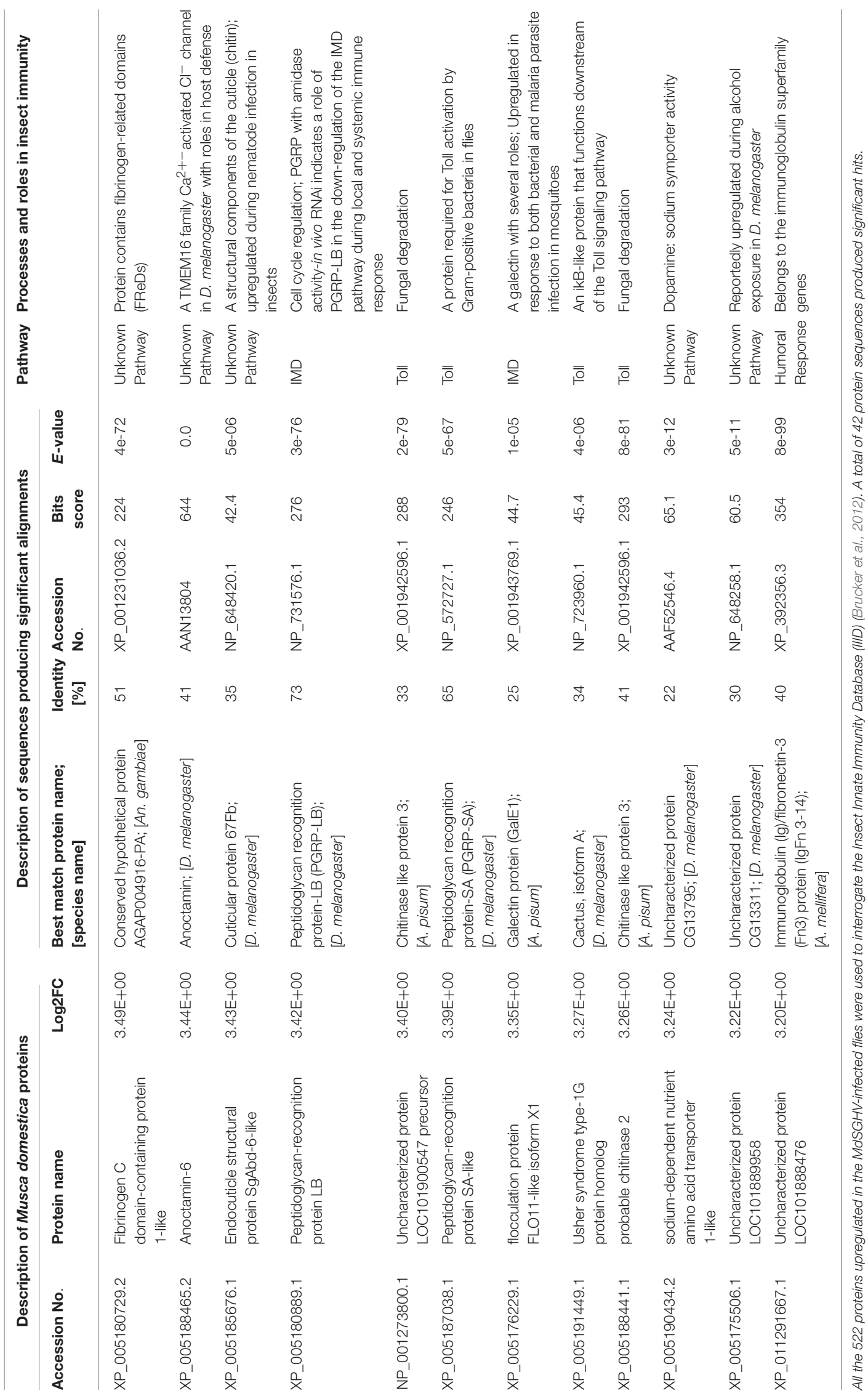




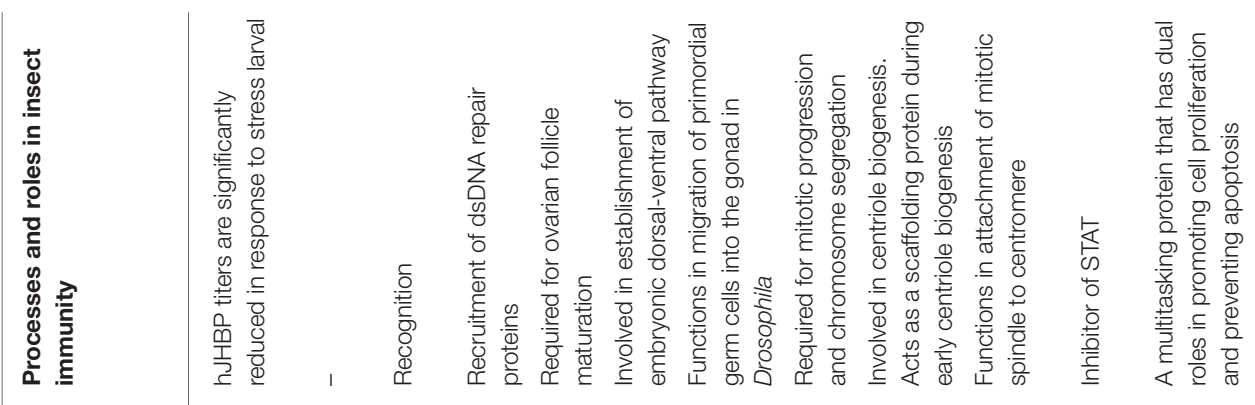

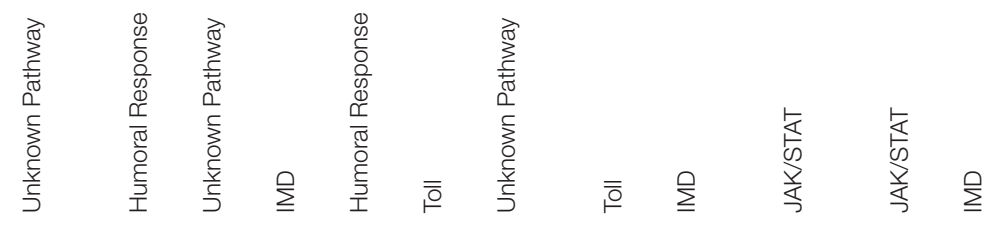

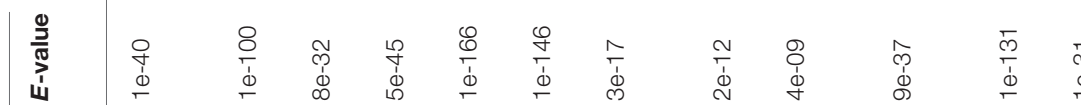

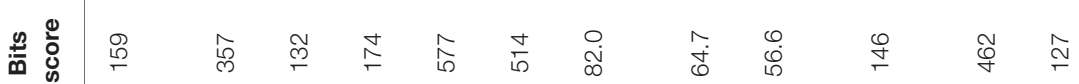

岁 旁

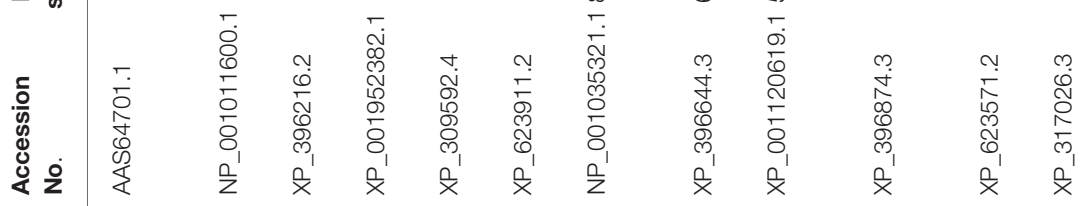

害宁

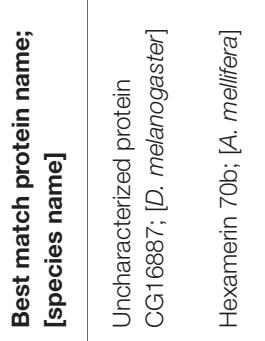

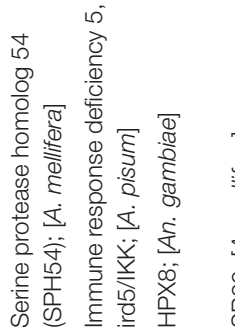

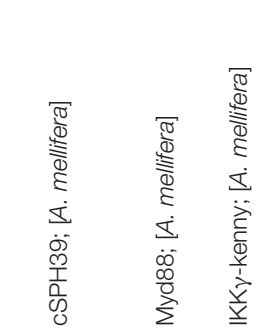

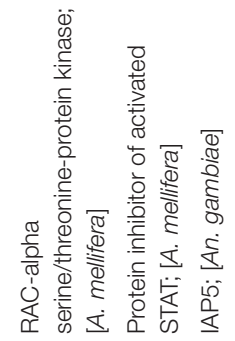

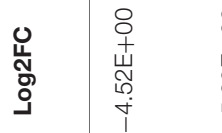

8
+
$\dot{+}$
0
0
$\dot{p}$
$\dot{p}$

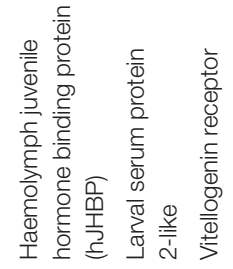

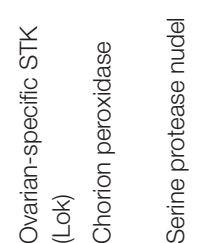

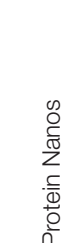

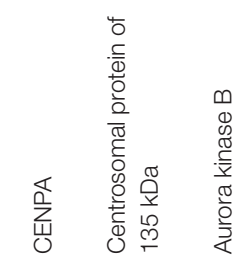

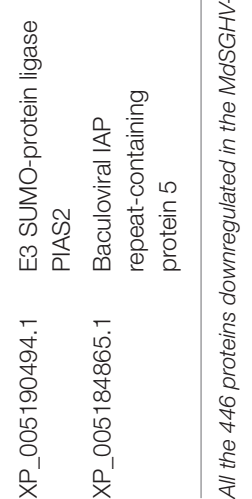




\section{Hemolymph Levels of Sesquiterpenes in Viremic and Healthy Flies}

Chemical analysis of the hemolymph extracts from PBS- and MdSGHV-injected females revealed fluctuations in titers of MF, JH-III, and JH-IIIB over the 4-day sampling period. In all healthy females, ovarian development reached either stage 3 (advanced vitellogenesis) or stage 4 (fully developed eggs) at 4 days. In these flies, hemolymph MF levels correlated with ovarian development and increased more than 20 -fold during 4 days post exposure to adult food (Figure 5). JH-III levels remained relatively low (0.3-2.0 $\mathrm{pg} / \mu \mathrm{l}$ hemolymph) throughout the sampling period. JHIIIB levels remained constant (2.5-3.5 pg/ $\mu$ l hemolymph) for the initial 3 days, but increased to $8.5 \mathrm{pg} / \mu \mathrm{l}$ hemolymph on day 4. MdSGHV infection completely shut down egg development; ovaries from viremic flies remained in the pre-vitellogenesis stage (stage 1). The JH-III and JH-IIIB levels were lower in the viremic than in healthy females throughout the 2-4 d-pi interval. The largest difference in hormone titers was observed in the MF; after $3 \mathrm{~d}$-pi, the hemolymph MF levels in viremic flies were 4to 10 -fold less than those detected in healthy flies.

\section{Impacts of Hormone Delivery on Viremic Female Flies}

The injection of the PBS-control or virus-injected female flies with either the peanut oil/acetone or the $10 \%$ ethanol carriers resulted in $\sim 10$ and $30 \%$ mortality, respectively, of flies within the $2 \mathrm{~d}$-pi of the carrier (data not shown). Injection of either carrier into PBS-injected females did not impact ovarian/egg development in survivors. Likewise, the survivors of PBS-injected females challenged with JH-III $(n=37), \operatorname{MF}(n=42)$, ecdysone $(n=35)$, or the hormone mix had normal ovarian development ranked at stage 4 at $5 \mathrm{~d}$-pi. Hormone challenges with JH-III ( $n=$ $34), \operatorname{MF}(n=41)$, or ecdysone $(n=36)$ into virus-challenged females did not alter SGH development; at $5 \mathrm{~d}$-pi, all females had SGH symptoms equivalent to virus controls. Furthermore, all surviving females of the hormone + virus treatments displayed ovarian stage 1 development identical to that observed in the virus control females at $5 \mathrm{~d}$-pi.

The impact of ecdysone treatments on modulating the transcription of the two female-specific genes (Hex2 and Yo2) was examined using established qPCR protocols (Lietze et al., 2007). Three d-pi (which equals 3 days on a protein-containing diet), the transcription of hexamerin and yolk protein was reduced 13- and 41-fold, respectively, in protein-fed viremic females when compared with protein-fed healthy females. In sugar-fed healthy females, a 7 - and 4 -fold reduction of hexamerin and yolk protein transcription was detected when compared with protein-fed healthy females. Injection of ecdysone stimulated both hexamerin and yolk protein transcription in both the sugar-fed and the virus-infected females in an expected, dosedependent manner (Table 4). Ecdysone amendments induced higher hexamerin transcript levels than the yolk protein transcript levels. When compared with baseline expression levels (1.00) for protein-fed healthy females, the $1 \mu \mathrm{g}$ ecdysone dose induced a $\sim 10.3$ - to 9.6-fold upregulation of hexamerin, and a $\sim 1.9$ - to 1.2 -fold upregulation of yolk protein in sugar-fed and virus-infected females, respectively. It should be noted that the ecdysone-induced rescue of these female-specific protein transcripts did not result in egg production in viremic flies.

\section{Impacts of SGHV on AMP Levels and on Insect-Associated Microbiota}

Both the RNA-Seq data and subsequent RT-qPCR validation assays confirmed that the transcript levels of AMPs were increased by MdSGHV infection; AMPs such as sarcotoxins, defensins, and attacins comprised many of the most highly upregulated genes (Table 2, Figure 3). As expected, insect homogenates prepared from healthy females contained no detectable activity targeting E. coli D31 but did contain constitutive lysozyme activity that targeted M. luteus. Injections of E. coli D31 (positive controls) into females induced antibacterial activity against $E$. coli D31; the relative lysozyme activity was similar to control treatments. Injection of the PBS healthy gland homogenate into female flies induced an immediate anti-D31 activity; however, this antibacterial activity was not sustained and was undetectable by $72 \mathrm{~h}$-pi. Unlike the healthy SG preparations, injection of the MdSGHV SG homogenates induced a sustained antibacterial (D31) activity throughout the 10-day sampling period. Lysozyme activity as measured by the $M$. luteus assay was suppressed initially by the MdSGHV challenge. Insect homogenates prepared from all of the above treatments had no detectable inhibitory activity against either Serratia marcescens or S. cerevisiae (data not shown). The induction of the innate defense systems by MdSGHV infection and the subsequent increase in the production of antibacterial activity did not appear to impact the cultivable microbiome associated with $M$. domestica. The estimates of CFUs associated with healthy flies $(n=32)$ ranged from $1.5 \times 10^{5}$ to $1.85 \times 10^{6}$ CFUs per fly. The average number of CFUs for both healthy (5.8 $\pm 4.9 \times 10^{5}$ CFUs per fly) and PBS-injected flies $(n=15,8.0 \pm 8.8$ $\times 10^{5}$ CFUs per fly) was not altered by MdSGHV infection at 72 h-pi. The average number of CFUs from viremic females $(n=24)$ was estimated to be $5.9 \pm 8.8 \times 10^{5}$ CFUs per fly. Likewise, the $\mathrm{CFU}$ estimates at $96 \mathrm{~h}$-pi were not impacted by virus infection.

\section{DISCUSSION}

\section{Expression of MdSGHV Transcripts}

Mapping RNA-Seq reads of the infected libraries onto the MdSGHV genome demonstrated a gradient in the predicted transcription rate of the viral ORFs. Significantly, the RNASeq approach proved to be highly sensitive; it detected seven putative ORFs $(7,9,19,39,41,59,80$, and 106,) undetectable previously by $3^{\prime}$ RACE and qPCR (Salem et al., 2009) and eight ORFs $(5,6,8,9,59,60,70$, and 106) undetectable in 454 sequencing of a cDNA library from viremic $M$. domestica (unpublished data, Table S7). In general, the transcript frequency of viral ORFs predicted by the RNA-Seq data correlated $\left(R^{2}=0.702\right)$ with transcript abundance predicted prior to the 454 reads (Figure S1B). However, predicted transcript frequencies were not associated with known structural features such as ORF position, tandem orientation, $3^{\prime}$-UTR structure, cleavage site, polyadenylation signals, etc., (see Table 1; Salem et al., 2009). 


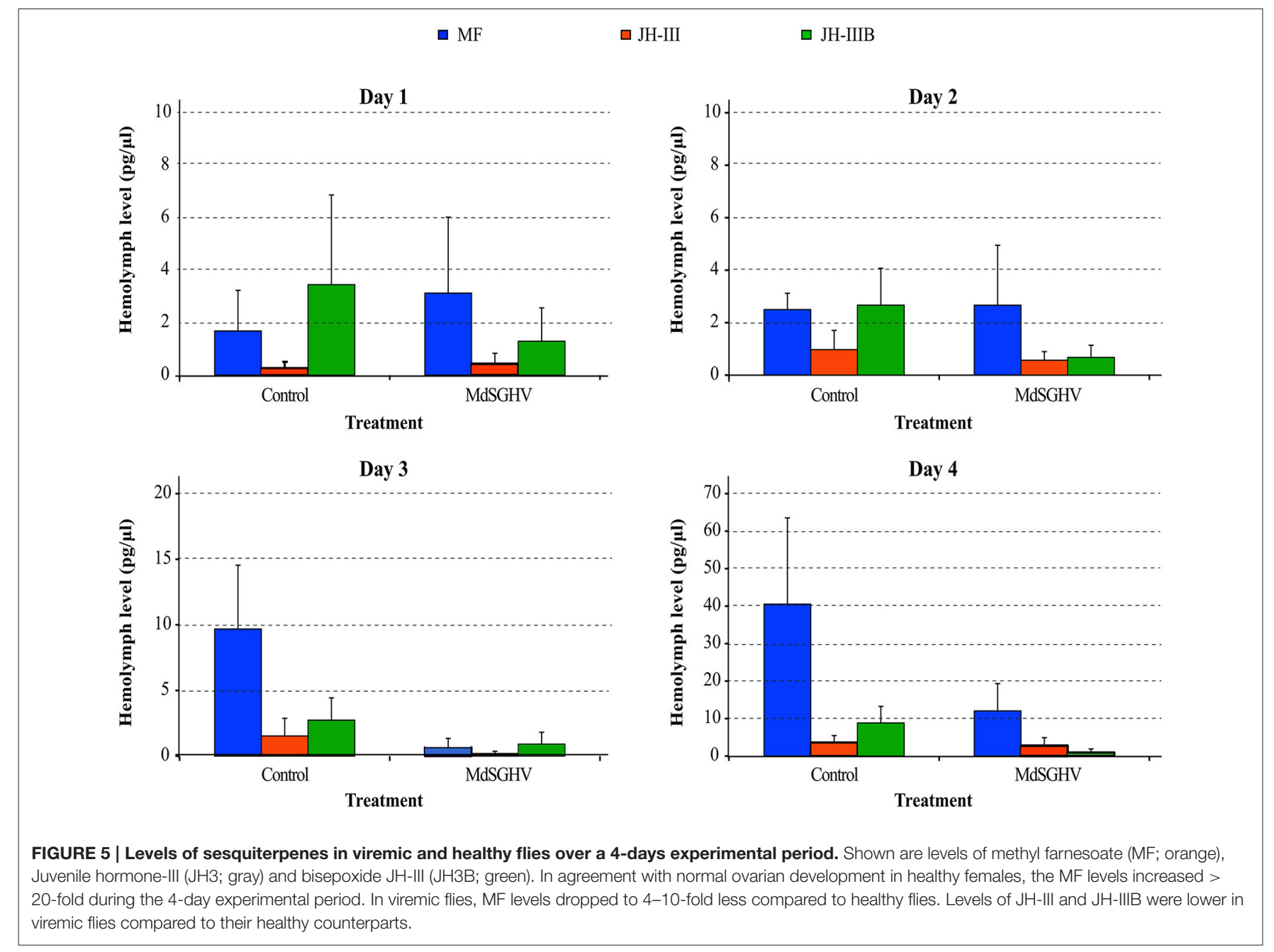

TABLE 4 | Impacts of ecdysone treatment on modulation of hexamerin-2 (Hex2) and yolk-2 (Yo2) gene expression in healthy and viremic M. domestica females.

\begin{tabular}{lcccc}
\hline Infection status & Diet & $\begin{array}{c}\text { Ecdysone } \\
\text { dose }[\boldsymbol{\mu} \mathbf{g} / \boldsymbol{\mu l}]\end{array}$ & \multicolumn{2}{c}{ Relative expression value } \\
\cline { 4 - 5 } & & & Hexamerin & Yolk protein \\
\hline Healthy & Sugar & 0 & 0.15 & 0.28 \\
Healthy & Sugar & 0.1 & 0.49 & 0.73 \\
Healthy & Sugar & 1.0 & 10.27 & 1.87 \\
Healthy & Protein & 0 & 1.00 & 1.00 \\
Viremic & Protein & 0 & 0.09 & 0.02 \\
Viremic & Protein & 0.1 & 0.10 & 0.06 \\
Viremic & Protein & 1.0 & 9.58 & 1.17 \\
\hline
\end{tabular}

The experimental flies were subjected to a 3-day sugar or protein diet and challenged with different ecdysone dosages.

However, low-copy ORFs $(5,6,7,8,9,69$, and 60) were clustered in the vicinity of direct-repeat (dr) regions (Figure 1).

Ten of the 15 highly abundant reads detected at $48 \mathrm{~h}$ pi mapped to ORFs encoding for structural peptides (GarciaMaruniak et al., 2008) (Figure 2; Table S2). These include ORF
96, encoding for the major viral envelope peptide (Boucias et al., 2013a), and ORF 47, a homolog to the occlusion-derived virus envelop protein 66 (ODV-E66), a component of the baculovirus envelope that is important for virus morphogenesis. Notably, odv-e66 encodes a non-secreted hyaluronate lyaselike enzyme that degrades hyaluronan, a component of the extracellular matrix (Vigdorovich et al., 2007); it is possible that this protein facilitates MdSGHV invasion (i.e., penetration) of host cells. Our annotations using various databases revealed that the MdSGHV086 is a putative viral matrix protein (Table S2). Immuno-cytochemical staining localized the MdSGHV086 protein on the external surface of the nucleocapsids exiting the nuclear pores of infected SG cells (Figure S2). Such viral matrix proteins provide the linkage between the viral envelope and nuclear-core components; they are found in many enveloped viruses (Battisti et al., 2012) and are crucial for viral assembly and budding. The remaining ORFs coding for highly abundant structural ORFs included 40,71, 85, 23, 28, 97, and 16 (Figure 2); these proteins remained without annotations, except for ORFs 16 and 85, which contained transmembrane domains (Table S2). Taken together, the high number of structural transcripts suggests that at $48 \mathrm{~h}$-pi, viral morphogenesis is well underway, a finding 
supported by the detection of infectious virus in the salivary secretions of viremic flies at $48 \mathrm{~h}$-pi (Lietze et al., 2009).

The remaining five of the 15 most-abundant proteins are non-structural proteins, of which the most abundant was ORF037, a homolog to a small, nuclear RNA-activating, complex protein involved in TATA box recognition (Table S2). Other non-structural proteins included MdSGHV048, a homolog to ring-infected erythrocyte surface antigen (a virulence factor protein), and MdSGHV082, a site-specific recombinase (RCI)-like protein that possesses a DNA-breaking and rejoining domain (Alberts, 2003). ORFs 92 and 93 remained without annotations. Other non-structural viral transcripts, which were detected in tissues that did not support viral replication/morphogenesis (Lietze et al., 2011b) and whose relative abundance generally mirrored the RNA-Seq results, included ORFs 1, 10, and 108 (Table S2). MdSGHV010 is homologous to the nudivirus OrNV mitochondrial carrier protein, a protein central to the transport of dATP and dTTP. Mimivirus uses this protein to target the host mitochondria as a source of dNTPs for its replication (Monné et al., 2007). MdSGHV001 is a DNApol-B delta subfamily homolog, which plays a central role in viral genome replication and transcription (Choi, 2012). MdSGHV106 is a PIF-3 homolog, an ODV-specific protein speculated to mediate nucleocapsid translocation along microvilli (Song et al., 2016) and thus facilitate release of nucleocapsids into the cell cytosol to initiate infection. We also quantified expression of ORF MdSGHV108, which encodes a homolog to the baculovirus Ac81-like protein. Ac81 is a lateexpressed, non-structural protein of BmNPV, which is thought to interact with the host cellular protein actin A3 (Chen et al., 2007) to facilitate intracellular transport of viral particles and infection.

\section{Putative Functional SGHV Proteins}

A comprehensive proteogenomic analysis (RNA-Seq and LCMS/MS) of the two SGHV strains (GpSGHV-Uga and GpSGHVEth) infecting the tsetse fly (Abd-Alla et al., 2016) revealed that 60 ORFs encode functional proteins, i.e., the ORFs contained TATAbox/poly(A) signals, had both transcript and peptide mapping, and/or had the G/T/ATAAG late-promoter motifs. Twenty of the MdSGHV proteins had significant homologies to the GpSGHV functional ORFs, 11 of which had RNA-Seq reads of $>10,000$ (Figure 1; Table S2). These included ORFs 4 (virion protein SGHV082), 13 (nucleocapsid protein SGHV083), 25 (vesicleassociated membrane protein), 29 (PIF-1), 30 (nucleocapsid protein), 33 (cell division protein 48), 55 (casein kinase isoform1D), 70 (LEF-8), 73 (nucleocapsid protein), 83 (LEF-3), and 84 (glutathione-S-transferase). The remaining nine ORFs had RNASeq reads ranging from 4,000 to 10,000 . The nine ORFs were: 12 (thymidylate synthase), 36 (MP-NASE), 39 (P74), 74 (LEF-9), 87 (LEF4), 89 (PIF-2), 102 (FAD dependent sulfhydryl oxidase), 107 (ABC transporter), and 108 (Ac81) (Figure 1; Table S2).

\section{Impacts of MdSGHV Infection on Host Transcriptome}

The majority of research conducted on insect DNA virus-host transcriptome interactions has involved the infection studies of susceptible cell lines challenged with high baculovirus titers. In such cases, the virus infection initiates a global shutdown of host transcription (Nobiron et al., 2003; Katsuma et al., 2007). However, studies conducted on host animals do not typically display this event; instead, viral infection displaying tissuespecific tropism occurs in a limited number of host cells. In the case of MdSGHV, viral infection at $48 \mathrm{~h}$-pi downregulated the transcription levels of 1,196 and 2,708 genes at the padj values of 0.01 and 0.2 , respectively, out of a total of 17,034 unigenes detected by RNA-Seq. MdSGHV displays a narrow tissue tropism, undergoing detectable levels of morphogenesis in selected gland tissues such as SGs and CA/CC (Lietze et al., 2011b). Prior qPCR analyses demonstrated the presence of viral transcripts and genome copies in samples derived from multiple housefly tissues. Further, electron microscopy revealed the presence of enveloped virus in the cytoplasm of tracheal cells. Here, it should be noted that the tracheal system is integrated into all insect tissues/cells except for the circulating hemocytes. This finding may explain the distribution of MdSGHV genome copies in tissues that do not support viral morphogenesis.

\section{Regulation of Genes Related to Virus-Induced Pathologies}

To maximize production of viral progeny and to evade/interfere with the host's immune and anti-viral stress responses, DNA viruses dampen the expression of host cell proteins via several strategies (Herbert and Nag, 2016). One strategy is co-transcriptional (in the nucleus) downregulation of the transcription of the host mRNAs such that the host's transcription factors and RNA polymerase complex components are availed for viral replication. In this strategy, viruses may encode proteases (to degrade) or other proteins (to inhibit) transcription factors of the host insect. From our study, the housefly transcription initiation factor II D (TFIID) was among the downregulated proteins $(\log 2 \mathrm{FC}=-6.0)$ (Table S3). Interestingly, MdSGHV037, which had the most RNA reads (See Figures 1, 2), encodes a homolog to the small nuclear RNA activating protein 190 (SNAP190), a TATA-box binding protein that may preferentially lead to transcription of viral genes. Probably this protein out-competes the downregulated housefly TFIID such that MdSGHV genes are preferentially transcribed by the host machinery.

Viruses also maximize their replication post-transcriptionally (in the nucleus or cytoplasm) via decapping or degradation of host mRNAs (Narayanan and Makino, 2013) and via interfering with mRNA splicing and nuclear export. The virally encoded decapping enzymes are usually expressed during early and late stages of viral infection to target host mRNAs such that host translation machinery is available almost exclusively for translation of viral RNAs (Liu et al., 2015). However, none of the MdSGHV genes had any significant homologies to known viral decapping enzymes. Alternatively, to promote replication, dsDNA viruses encode their own capping enzymes to protect their mRNAs (Decroly et al., 2012). The highly transcribed MdSGHV087 is a LEF-4-like protein (Figures 1, 2; Table S2), which is putatively functional in GpSGHV (Abd-Alla et al., 2016); 
LEF-4 is an mRNA-capping protein essential for baculovirus replication (Jin et al., 1998).

Nuclear replicating DNA viruses must deliver/export their genomes into and out of the host cell nucleus, a process orchestrated via the tightly regulated traffic through the nuclear pore complex (NPC) (Fay and Panté, 2015). Depending on the virus, the nuclear transmission of nucleocapsids involves specific transport mechanisms to import/export the viral genome (and associated proteins) through the NPC. To allow traffic of the viral nucleoprotein complexes across the NPC, one would expect degradation of nuclear pore proteins and removal of the nuclear basket that controls active NPC functions. Some viruses induce activation of nuclear factor NF- $-\mathrm{B}$, which in turn results in auto-activation of cellular caspases that promote viral nucleoprotein complex export (Mühlbauer et al., 2015). Prime caspase substrates include nuclear pore complex protein-153 (Nup153) and lamins (Fischer et al., 2003), two important NPC nuclear basket components and key players in nuclear import/export control. This potentially accounts for the upregulation of caspases-3/-8, lamins, and NF- $\kappa-B$ p110 subunits, as well as the downregulation of Nup153 in MdSGHV-infected samples (see Table S4).

To limit viral replication and protect the host's genome, infected cells express components of DNA damage response (DDR) pathways, which are responsible for detection and repair of DNA lesions; DDR ultimately induces apoptosis (Weitzman et al., 2010). This possibly partially explains the modulation of proteins of the phosphatidylinositol signaling system in the MdSGHV samples (see Tables S5, S6). Some viruses respond by synthesizing anti-apoptotic proteins that allow persistent viral infections. In addition to conferring selective advantages to the virus, viral interference with apoptosis plays essential roles in cellular transformations such that the cells not only survive but also simultaneously grow and efficiently produce the progeny virus (Thomson, 2001). It is therefore noteworthy that MdSGHV078 encodes (moderately; Figure 2) IAP, a potent and specific inhibitor of caspase- 3 capable of reversing mitochondrial membrane permeabilization (MMP) (Crook et al., 1993); MMP is a key event in the induction of apoptosis.

Upon activation, DDR-associated kinases phosphorylate multiple substrates, including histones and chromatinremodeling complexes (Van Attikum and Gasser, 2009). Ultimately, the DDR signaling results in cell-cycle arrest that allows either DNA repair or apoptosis. The virus-induced blockade of apoptosis, coupled with enhancement of virus replication and cellular transformations, undoubtedly not only promotes host genomic instability but also contributes to viral pathogenesis. DNA viruses are able to disable some antiviral facets of the DDR (Weitzman et al., 2010), including repression of histone/chromatin modifications, to render the host cells more favorable to viral DNA replication. This probably explains the downregulation of at least six components of the forkhead box O (FoxO) signaling pathway in the MdSGHVinfected samples, including the serine/threonine-protein kinase ATM (a DDR) (See Table S5). It should be noted that since FoxO is primarily responsible for the maintenance of cellular metabolic stress (Eijkelenboom and Burgering, 2013), FoxO may engage apoptosis if the virus-induced cellular damages become excessive. FoxO is also implicated in epigenetic regulation of gene expression (Calnan and Brunet, 2008).

Under the stress of virus infections, cells must maintain normal homeostasis; otherwise, they die. The main homeostatic adaptive cellular responses are hypertrophy (enlarged cells incapable of dividing), hyperplasia (enlarged cells capable of replication), atrophy (shrinkage) and metaplasia (reversible replacement of an adult cell type with another). To accommodate the additional functional demands for replication of viral progenies, the MdSGHV induces both nuclear and cellular hypertrophy, resulting in a non-lytic increase in individual cell sizes (without cell number increase), and ultimately, enlarges infected glands. Alternatively, the GpSGHV, which causes limited cellular hypertrophy, induces cellular hyperplasia of the SG cells (Kariithi et al., 2013).

\section{MdSGHV-Induced Female Sterility}

The major outcome of MdSGHV infection is female sterility, i.e., inhibition of ovarian development in young flies and subsequent gonadotropic cycles in older females (Lietze et al., 2008). RTqPCR and present RNA-Seq data clearly showed that virus infection blocks production of the female-specific yolk proteins (YPs) and female hexamerin, which serve as the nutritional basis for egg development (Moreira et al., 2009; Siegenthaler et al., 2010). The YPs synthesized in the fat body are released into the hemolymph and sequestered by the developing basal oocytes for subsequent yolk formation (Dong et al., 2009). In the RNA-Seq experiments, the virus-induced suppression of these female-specific gene transcript levels was not as pronounced as had been found in an earlier experiment, when at 3 days postinfection, viremic flies contained 94-fold and 1,596-fold fewer hexamerin and yolk protein transcripts, respectively, than did control flies (Lietze et al., 2008). A possible explanation may be due to sampling, because hexamerin and YP production fluctuate during the gonadotropic cycle, or to variance between the RTqPCR and RNA-Seq analyses.

In dipterans, oogenesis is regulated by a hormonal regime (sesquiterpenoids and steroids) that is initiated when emerging flies ingest a protein meal whereby a pulse of $\mathrm{JH}$ makes the fat body competent for YP synthesis (Agui et al., 1985). The JH synthesis, involving 13 discrete enzymatic steps (Noriega, 2014) takes place in the CA. Our results gave evidence that the housefly CA/CC supports limited MdSGHV morphogenesis (see Figure S3). In houseflies, JH positively regulates YP levels; allatectomy abolishes YP production (Adams et al., 1985). Our RNA-Seq data sets did not provide clear evidence of blockage of the $\mathrm{JH}$ pathway; rather, several of the $\mathrm{JH}$ enzymes were variously but insignificantly modulated in the MdSGHV-infected flies. However, chemical analysis of hemolymph revealed that sequesterpenoid levels in viremic females were lower than those detected in healthy flies. Whether or not these differences have a physiological impact on modulating vitellogenesis is unknown. However, amending virus-infected females with exogenous hormone treatments (including JH-III, MF) did not result in normal oogenesis, suggesting that the observed reproductive block was not due solely to a lack of sesquiterpenoids. 
An alternative target may be downstream of the $\mathrm{JH}$ biosynthetic pathway. The JH-resistant Methoprene-tolerant (Met) gene detected in the red flour beetle $T$. castaneum (Konopova and Jindra, 2007) is believed to play a critical role in insect morphogenesis. It is thought that $\mathrm{JH}$ is an activating ligand for Met (Jindra et al., 2013). Met requires its interacting partner Taiman (Tai) for proper functioning. In mosquitoes, JH acts via Met/Tai to regulate expression dynamics of thousands of genes during female reproduction (Zou et al., 2013). Interestingly, both a homolog to the Drosophila Met gene was downregulated $(\log 2 \mathrm{FC}=-1.9)$ and several Drosophila Tai homologs were moderately downregulated $(\log 2 \mathrm{FC}=-1.7$ and -0.9) in MdSGHV-infected flies.

It should be emphasized that in $M$. domestica, the main controlling agent of $\mathrm{YP}$ synthesis is 20-E pulse, which is produced by the ovarian follicular epithelium (Adams et al., 1985). Cytochrome P450 (CYP450) enzymes are involved in the ecdysteroid metabolic pathways (Iga and Kataoka, 2012) and are transcriptionally regulated to support the high biosynthetic activities occurring during ecdysteroid-mediated pulses that trigger molting (Rewitz et al., 2006). In the current study, several components of the CYP450 transcripts were variously modulated in the MdSGHV-infected samples. These included CYP304a1, 307a1, 313a4, 18a1, 28d1, CYP12a2-like, 9f2, 6d1like, 4e2-like, 6a14, and 6a21. The levels of these gene transcripts are responsible for regulating the ecdysone titer. The downregulated CYP307a1 (Spook), responsible for converting 22, 2-dideoxyecdysone to 2-deoxyecdysone, is a stage-specific regulator for ecdysteroid synthesis in insects (Gilbert, 2004; Ono et al., 2006), whereas others, such as CYP18a1, are implicated in the inactivation of 20-E. Hormone treatment of viremic females with exogenous ecdysone did upregulate the transcription of female-specific genes (Yp, hexamerin) but did not result in normal egg development.

\section{MdSGHV-Induced Female Behavior Changes}

In addition to shutting down oogenesis, MdSGHV infection also causes perturbation in housefly mating behavior, specifically the refusal of infected females to mate with either healthy or viremic male flies. In healthy virgin flies, mating behavior is a fixed pattern, initiated by males who mount and stroke/caress the female, stimulating her to extend her ovipositor into the male genital opening, resulting in a copulation event lasting for about $60 \mathrm{~min}$ (Murvosh et al., 1964). Infected females, however, respond to male courtship but refuse to extend their ovipositor, thus terminating the mating sequence. From our RNA-Seq data, homologs to the Drosophila sex lethal (Sx1), fruitless (Fru), dissatisfied (Dsf), transformer-2 (Tra-2), and doublesex (Dsx), were detected, but only Tra-2 $(\log 2 \mathrm{FC}=$ -1.00 ) had a padj value of $<0.01$. However, the conclusions that can be drawn from these data are limited; transcripts of these genes could be better resolved by analyses of tissuespecific RNA-Seq libraries. This qualification notwithstanding, the insect's mating behaviors are also controlled by other hormones, including sesquiterpenoids. In fact, it has long been established that receptivity of virgin males for mating is under the control of JH; Loher and Huber (1966) reported that removal of corpora allata from newly-eclosed grasshopper females resulted in sustained active rejection of courting males. On the other hand, by injecting $\mathrm{JH}$, the authors were able to induce the allatectomized females to accept courting males. It also has been demonstrated that rising hemolymph $\mathrm{JH}$ titers stimulate other behavioral traits, including host-seeking in newly-eclosed mosquitoes. For instance, it has been reported that $\mathrm{JH}$ activates Met protein in the formation of a transcription repressor complex with proteins Hairy and Groucho; the interactions between these proteins is essential for female maturation (Jindra, 2016), and consequently influences the mating behavior. In our RNA-Seq data, we detected a Drosophila homolog to protein Groucho, which was downregulated, albeit at low level $(\log 2 \mathrm{FC}=-0.5)$. On the other hand, protein Hairy, which is downstream protein Met, was slightly upregulated in our study $(\log 2 \mathrm{FC}=0.2)$.

\section{Impact of MdSGHV Infections on Housefly Innate Defense Systems}

Insects have three main antiviral defense mechanisms, one of which is the first antiviral defense line mediated by the siRNA arm of the RNAi pathways (Ding, 2010). Insects encode dicer-1 (Dcr-1) and Dcr-2 to generate and activate siRNAs, respectively; Dcr-2 directly links the siRNA pathway to the Jak/STAT pathway (via the siRNAs) (Paradkar et al., 2012). Argonaute-2 (Ago-2) executes siRNA-mediated gene silencing (Okamura et al., 2004). Interestingly, Ago1 was downregulated by MdSGHV infection; Ago-1 is important in production of miRNAs that are crucial to oogenesis (Nakahara et al., 2005; Azzam et al., 2012). Disruption of LmAgo- 1 by RNAi dramatically decreased vitellogenin transcription and severely impaired follicular epithelium development, terminal oocyte maturation, and ovarian growth, supporting the notion that Ago1-dependent miRNAs are required for $\mathrm{JH}$-mediated vitellogenesis and egg production in locusts (Song et al., 2013). From our RNA-Seq data, a homolog to the Drosophila Dcr-2 was upregulated (log2FC $=2.9)$, as well as two Ago-2 homologs $(\log 2 \mathrm{FC}=2.1$ and 3.9$)$ in infected flies, implying that siRNA pathway is an important antiviral immune response in houseflies. However, the RNASeq did not provide any evidence that MdSGHV encodes viral suppressors of siRNA. Potentially, MdSGHV could have evolved other RNAi counter-defense mechanisms, such as sequestration in cytoplasmic compartments, which may be inaccessible to the host's RNAi machinery.

The siRNA antiviral response does not necessarily clear viral infections, which brings into play the innate antimicrobial (Jak/STAT) and NF- $\kappa-\mathrm{B}$ innate immune pathways (Imd and Toll) (Kingsolver et al., 2013). One of the Jak/STAT pathway components, which was moderately downregulated $(\log 2 \mathrm{FC}$ $=-1.2$ ) in MdSGHV-infected flies, is tyrosine-protein kinase hopscotch. Hopscotch transphosphorylates STATs, which are subsequently transported to the nucleus to regulate expression of downstream effectors of the pathway. Perhaps downregulation of hopscotch could be a strategy of MdSGHV to disrupt the phosphorylation and/or nuclear export of STATs. The 
Jak/STAT pathway ligand, Vago protein (Paradkar et al., 2012), whose expression is Dcr-2-dependent, was moderately upregulated $(\log 2 \mathrm{FC}=1.5)$ in the infected flies. Moreover, the virus-induced stress in insects results in upregulation of other Jak/STAT downstream effectors such as the thioestercontaining proteins (Teps), which mediate phagocytotic antiviral responses (Levashina et al., 2001). Notably, our RNA-Seq revealed various levels of upregulation $(\log 2 \mathrm{FC}$ of between 1.1 and 3.4) of Drosophila and Glossina Tep-1, 2 and -4 homologs. In terms of the NF- $-\mathrm{B}$, the Imd and Toll activate cellular transcription mediated by Relish and NF-к-B orthologs (the terminal transcription factors for Imd) and Dorsal/Dorsalrelated proteins (Dif; for the Toll pathway). The two isoforms of the housefly NF-kappa-B p110, which were upregulated in MdSGHV-infected flies ( $\log 2 \mathrm{FC}=1.6)$, are Drosophila Relish homologs. The two Toll transcription factors also are centrally involved in early embryogenesis, which possibly explains the moderate downregulation of Dorsal homologs in the infected flies, including two isoforms of embryonic polarity protein dorsal-like protein $(\log 2 \mathrm{FC}=-2.4$ and -0.9$)$ and dorsalinteracting protein 3 (Dip3; $\log 2 \mathrm{FC}=-2.1$ ). We also noted upregulation of various components of Toll pathway signaling in the infected flies, including PGRP-SD/SA, GNBP-3, and Cactus.

An alternative antiviral defense mechanism is the autophagy pathway, a highly conserved cellular degradative pathway, which is induced by among others ER stress, ROS and mitochondrial damage triggered by pathogen infection (Chiramel et al., 2013). Autophagy is independent of the Jak/STAT, Imd and Toll pathways, and is implicated in the elimination of intracellular pathogens (Yano et al., 2008; Nakamoto et al., 2012). As an antiviral machinery, the autophagy system, which is mediated via autophagy-related genes (Atgs), not only directly degrades viral particles (autophagosomal degradation), but also delivers viral components to Toll receptors on endosomes (lysosomal degradation), and transports host's antiviral proteins to virus replication sites (to interfere with viral replication) (Yordy et al., 2013). Some of the key proteins involved in autophagy include microtubule-associated proteins (MAPs), $\gamma$-aminobutyric acid receptor-associated proteins (GABARAP). From out RNA-Seq data, Map-2-like and Gabarap-like genes were moderately upregulated $(\log 2 \mathrm{FC}=2.2$ and 3.8 , respectively) in viremic compared to healthy flies. In Drosophila, the autophagy pathway is mediated via the phosphotidylinositide-3-kinase (PI3)/Atk/mTOR pathway; knock-down of autophagy-related genes increased virus production in both Drosophila S2 cells and in the flies (Shelly et al., 2009). From our RNA-Seq data, Drosophila $\mathrm{PI} 3 \mathrm{~K} / \mathrm{mTOR}$ homologs were slightly downregulated in the MdSGHV-infected flies, including PI3K59F $(\log 2 \mathrm{FC}=$ $-0.2)$, Pi3K68D $(\log 2 \mathrm{FC}=-0.3),(\log 2 \mathrm{FC}=-0.4),(\log 2 \mathrm{FC}=$ $-0.6)$ and an mTOR-like protein $(\log 2 \mathrm{FC}=0.1)$. Further, it has been shown that some dsDNA viruses have over evolutionary time developed tactics not only to evade, but also to exploit the autophagy system to the advantage of viral replication (See reviewed in Chiramel et al., 2013). Various pathways such as the above-mentioned Akt/mTOR signaling pathways are involved (Shoji-Kawata and Levine, 2009).
A major outcome of MdSGHV infection is the higher transcription level of a cocktail of AMP genes, resulting in sustained increase in antibacterial cationic peptide activities. Complementing the cationic peptides is the increased transcription of various PGRPs and lectins, molecules that serve as sentinels in the non-self recognition of invasive bacteria and regulators of commensal gut bacteria (Royet et al., 2011). However, the increase in AMP and PGRP activities did not impact the cultivable microbiome associated with the host; total CFUs in viremic flies were comparable to those estimated for healthy flies. Here, it should be noted that the housefly microbiome is localized on the cuticle and in the lumen of the digestive tract; hence, the microbiome may not be exposed to AMPs affiliated with internal tissues. Furthermore, the ability of the microbiome to coexist with the house flies lab colony may be due intrinsic resistance to AMPs. Moreover, MdSGHV is non-lytic, replicates in limited tissues (SG, CA), and causes a chronic nonlethal disease. The induction of innate defenses by infection may underlie the tissue tropism of this virus and/or may serve to suppress secondary infections, thereby assuring the continued production and release of virus from viremic flies.

\section{CONCLUSIONS AND FUTURE PERSPECTIVES}

The RNA-Seq analysis demonstrated that the MdSGHV infection caused a massive alteration in host gene transcription. Active MdSGHV replication occurred at $48 \mathrm{~h}$-pi as evidenced by the abundant levels of MdSGHV transcripts. Annotation of viral genes was associated with various facets of viral morphogenesis, including host cell invasion/penetration, nucleocapsid cytoplasmic traffic, genome replication, assembly, SGHinduction (virulence factors), and egress (budding). The multiple fronts employed by MdSGHV, including downregulation of host transcription factors, epigenetic gene expression regulation, production of virally encoded capping enzymes and blockade of apoptosis, significantly contributes to SGH development in houseflies. It would be interesting to make a comparative analysis of Glossina-GpSGHV and Musca-MdSGHV models, especially to identify host factors involved in the development (or lack thereof) of diagnostic SGH symptoms.

MdSGHV transcription/infection caused a massive reduction of transcript levels of genes associated with female reproduction/egg development; these findings confirmed prior observations on MdSGHV-induced sterility. The gene product(s)/pathway(s) that is responsible for oogenesis shutdown is unclear. Our speculation was that limited infection of CA/CC-complex may alter hormonal titers required to upregulate vitellogenesis/egg production. Our hormone therapy attempts, although rescuing the transcription of female-specific proteins, did result in egg production. It is possible some of the "down regulated" genes such as the Ago-1 or Spook homologs may prevent the upregulation of the multiple genes/pathways involved in ovary/egg maturation. Potentially, the MdSGHVmediated modulation of hormones may explain the refusal 
of viremic females to mate; the CA/CC-produced hormones mediate not only insect morphogenesis, ovary maturation, and general physiology but also modulate mating behavior. A key to understanding the observed sterility is identifying the viral gene product(s) that triggers this event. However, it is not easy to unravel the roles of specific, virally encoded factors (proteins) at the molecular level; most MdSGHV proteins have no identifiable homologs. Potentially, testing recombinant viral gene products in female houseflies or expressing gene constructs in heterologous hosts such as Drosophila may elucidate function, setting the stage for the discovery of a novel viral-gene-based insect birth control. Additional studies should address the full repertoire of players downstream in the $\mathrm{JH}$ pathway.

MdSGHV infection resulted in the increased transcript levels of genes associated with innate defense pathways. One may speculate that the observed increase is induced by virus infection/replication. Limited bioassays have demonstrated that the translation of these genes results in increased levels of AMPs. The induction of these pathways may potentially provide these viremic insects protection against opportunistic pathogens or serve to restrict the infection to select glandular tissues. It is important, however, to note that the interpretations of the innate defense responses were complicated by the fact that the experiments were conducted on the whole animal and not a cell culture. The physiological status of the healthy female fly is dynamic; for instance, access to a protein meal is known to trigger a massive physiological switch that is directed at egg production (Attardo et al., 2005). It has been proposed that in insects, there are reproduction-immunity trade-offs in resource allocations (Schwenke et al., 2016); these trade-offs are mediated by the endocrine and metabolic signaling, which heavily rely on the fat body. Critical of these resources include yolk proteins, vitellogenins, lipid, RNAs and ribosomes, which are allocated intricately for egg production reduces immune response and alternatively the induction of the innate defense system decrease reproductive fitness. Therefore, many of the pathways that were apparently upregulated could be the result of MdSGHV infection to block reproduction, and thus, at $48 \mathrm{~h}$-pi, the viremic females are reminiscent of individuals that are more comparable to newly eclosed females.

\section{AUTHOR CONTRIBUTIONS}

DB conceived the study design, conducted the infection assays, RNA extractions, diagnosis, data compilation, and wrote various sections of the manuscript. HK performed gene annotation, pathway analysis, data compilation, integration of data sets, and wrote the major sections of the manuscript. XY conducted and analyzed the RT-qPCR reactions. FY conducted the compilation and initial annotation the RNA Seq data set. PT conducted sequesterpenoid HPLC analysis. CV conducted fly injections, RNA extractions, PCR reactions, antimicrobial bioassays and bacterial CFU determination. All the authors read and approved the final version of the manuscript.

\section{FUNDING}

This work was financially supported by a University of Florida Multistate Minigrant, and by the Joint FAO/IAEA (CRP \#D42015). Division of Nuclear Techniques in Food and Agriculture, Vienna, Austria.

\section{ACKNOWLEDGMENTS}

The authors thank S. Shanker (NextGen DNA Sequencing Core, UF ICBR), Verena Leitze (Entomology and Nematology Department, UF) and K. Kelly (UF Electron Microscopy Core, UF ICBR) for their technical support. The authors acknowledge C. Geden (USDA/ARS, Gainesville, FL) for providing fly pupae used in these experiments and F. Deng and $\mathrm{Z}$. Hu (Wuhan Institute of Virology, Wuhan, China) for providing the anti-ORF 86 antibody.

\section{SUPPLEMENTARY MATERIAL}

The Supplementary Material for this article can be found online at: http://journal.frontiersin.org/article/10.3389/fmicb. 2017.00583/full\#supplementary-material

The data discussed in this paper have been deposited in the NCBI's Gene Expression Omnibus (GEO) (Edgar et al., 2002), and are accessible through GEO Series accession number GSE88939 (https://www.ncbi.nlm.nih.gov/geo/query/ acc.cgi? acc $=$ GSE88939).

Figure S1 | Mapping of RNA-Seq reads onto the MdSGHV genome. The figure shows a regression analysis of the size and RNA-Seq read frequency of all the 108 ORFs in the MdSGHV genome (A) and a comparison between the frequency of the RNA-Seq reads and prior 454 data set (B) (see Table S7).

Figure S2 | Immuno-localization of MdSGHV086 using mono-specific anti-MdSGHV086 IgG. The antibody was generated in rabbits using an antigen-derived recombinant MdSGHV086. (A) Western blot probed with anti-MdSGHV086 antibody: lane 1 pre-stained MW standards, lane 2 healthy gland homogenate, lane 3 infected gland displaying SGH, and lane 4 gradient purified MdSGHV. (B) TEM micrographs of thin section of hypertrophied SGs probed with anti-MdSGHV086 antibody and a commercial anti-rabbit colloidal gold secondary probe. The MdSGHV086 was localized on the surface nucleocapsid being synthesized in the virogenic stroma of the infected nucleus. (C) Immuno-staining of nucleocapsids exiting through the nuclear pores of infected SG cells. (D) Enveloped virus in the gland lumen, note the antibody probe appeared to localize between the capsid and outer envelope region.

Figure S3 | TEM micrographs of the CA/CC complex dissected from infected females at 48h-pi. (A) Depicts a cell containing a hypertrophied nucleus with condensed chromatin reminiscent of that observed in infected SG cells. (B) Is a higher magnification of this region showing the presence of numerous elongate MdSGHV nucleocapsids providing evidence for limited viral morphogenesis in the CAVCC complex.

Table S1 | Description of host genes and PCR primer pairs. Primer sets designed from ORFs from Musca domestica genes that displayed varying degrees of regulation of MdSGHV infection.

Table S2 | Annotations of 108 proteins in the MdSGHV genome. Annotations of 108 proteins in the MdSGHV genome: Descriptions of the best BLASTp homologies to the known viral and/or cellular genes are indicated (Bit Score $\geq 40$; Expect value of E-6). The signature domains in the protein sequences are indicated in the last column. Amino acid coordinates of the $\mathrm{N}$-terminal signal peptide (SP) sequence and transmembrane domains (TM) are indicated in the last column. Of the 108 MdSGHV proteins, 31 proteins are 
putative transmembrane proteins as they contained at least one TM. ORFs marked with asterisks $(*)$ denote 10 of the most abundant MdSGHV structural proteins based on the RNA-Seq reads at $48 \mathrm{~h}$-pi (Compare with Figure 2). The 20 MdSGHV ORFs in bold encode proteins that ate homologous to proteins in the two SGHVs infecting the tsetse fly (GpSGHV-Uga and GpSGHV-Eth; Abd-Alla et al., 2016), which have been reported to be functional proteins [i.e., contain TATA-box, poly(A) signal sequences and have been confirmed by RNA-Seq and LC-MS/MS].

Table S3 | Gene Ontologies (GO) of downregulated host proteins in viremic houseflies. Annotations and description of the GO terms associated with the top 50 genes (padj $\leq 0.01$ ) in the housefly that were negatively regulated by MdSGHV infection at 48 pi. For the annotations, the translated protein sequences were initially blasted using BLASTp Blast2GO (Conesa et al., 2005). Further annotations were performed at the nr-NCBI database (Bitscore $\geq 80$; $E$-value $\leq 1.0 \mathrm{E}-6$ ) on Drosophilidae database. A total of 14 uncharacterized housefly sequences (marked with asterisks) yielding uncharacterized Drosophila homologs were assigned the closest characterized dipteran homologs available at the nr-NCBI database.

Table S4 | Gene Ontologies (GO) of upregulated host proteins in viremic houseflies. Top fifty genes having a padj value of $\geq 0.01$ positively regulated by MdSGHV infection at 48pi. Gene information derived from annotation to $M$. domestica or Drosophila databases. For the annotations, the translated protein sequences were initially blasted using BLASTp Blast2GO (Conesa et al., 2005).

\section{REFERENCES}

Abd-Alla, A. M., Kariithi, H. M., Cousserans, F., Parker, N. J., Ince, I. A., Scully, E. D., et al. (2016). Comprehensive annotation of Glossina pallidipes salivary gland hypertrophy virus from Ethiopian tsetse flies: a proteogenomics approach. J. Gen. Virol. 97, 1010-1031. doi: 10.1099/jgv.0.000409

Abd-Alla, A. M., Cousserans, F., Parker, A. G., Jehle, J. A., Parker, N. J., Vlak, J. M., et al. (2008). Genome analysis of a Glossina pallidipes salivary gland hypertrophy virus reveals a novel large double-stranded circular DNA virus. J. Virol. 82, 4595-4611. doi: 10.1128/JVI.02588-07

Abd-Alla, A. M., Kariithi, H. M., Parker, A. G., Robinson, A. S., Kiflom, M., Bergoin, M., et al. (2010). Dynamics of the salivary gland hypertrophy virus in laboratory colonies of Glossina pallidipes (Diptera: Glossinidae). Virus Res. 150, 103-110. doi: 10.1016/j.virusres.2010.03.001

Abd-Alla, A. M. M., Parker, A. G., Vreysen, M. J., and Bergoin, M. (2011). Tsetse salivary gland hypertrophy virus: hope or hindrance for tsetse control? PLoS Negl. Trop. Dis. 5:e1220. doi: 10.1371/journal.pntd.0001220

Abd-Alla, A. M., Vlak, J. M., Bergoin, M., Maruniak, J. E., Parker, A. G., Burand, J. P., et al. (2009). Hytrosaviridae: a proposal for classification and nomenclature of a new insect virus family. Arch. Virol. 154, 909-918. doi: 10.1007/s00705-009-0398-5

Adams, T. S., Hagedorn, H. H., and Wheelock, G. D. (1985). Haemolymph ecdysteroid in the housefly, Musca domestica, during oogenesis and its relationship with vitellogenin levels. J. Insect Physiol. 31, 91-97. doi: 10.1016/0022-1910(85)90013-7

Agui, N., Izumi, S., and Tomino, S. (1985). The role of ecdysteroids and juvenoids in vitellogenin levels and follicle development in the housefly, Musca domestica. Appl. Ent. Zool. (Japan) 20, 179-188.

Alberts, B. (2003). DNA replication and recombination. Nature 421, 431-435. doi: $10.1038 /$ nature 01407

Attardo, G. M., Hansen, I. A., and Raikhel, A. S. (2005). Nutritional regulation of vitellogenesis in mosquitoes: implications for anautogeny. Insect Biochem. Mol. Biol. 35, 661-675. doi: 10.1016/j.ibmb.2005.02.013

Azzam, G., Smibert, P., Lai, E. C., and Liu, J. L. (2012). Drosophila Argonaute 1 and its miRNA biogenesis partners are required for oocyte formation and germline cell division. Dev. Biol. 365, 384-394. doi: 10.1016/j.ydbio.2012. 03.005

Bagos, P. G., Liakopoulos, T. D., Spyropoulos, I. C., and Hamodrakas, S. J. (2004). PRED-TMBB: a web server for predicting the topology of ß-barrel outer membrane proteins. Nucleic Acids Res. 32, W400-W404. doi: $10.1093 / \mathrm{nar} / \mathrm{gkh} 417$
Further annotations were performed at the nr-NCBI database (Bitscore $\geq 80$; $E$-value $\leq 1.0 \mathrm{E}-6$ ) on Drosophilidae database. The seven uncharacterized housefly sequences (marked with asterisks) yielding uncharacterized Drosophila homologs were assigned the closest characterized dipteran homologs available at the nr-NCBI database. Five sequences remained without any significant hits to characterized proteins in the databases.

Table S5 | Pathways associated with upregulated proteins. KEGG pathway mapping of Musca domestica genes whose transcription was upregulated in the MdSGHV-infected flies compared to the PBS-injected controls. The numbers of the host genes that were upregulated are shown in reference to the total number of housefly genes that have been associated with each pathway. The KEGG pathways are hyperlinked.

Table S6 | Pathways associated with downregulated proteins. KEGG pathway mapping of Musca domestica genes whose transcription was downregulated in the MdSGHV-infected flies compared to the PBS-injected controls. The numbers of the host genes that were downregulated are shown in reference to the total number of housefly genes that have been associated with each pathway. The KEGG pathways are hyperlinked.

Table S7 | Comparative transcriptome analysis. Comparison of the frequency of RNA-Seq reads of the MdSGHV infected libraries (V1, V2, and V3) mapped to all the 108 ORFs in the viral genome to the previously 454 reads generated from cDNA generated from a total RNA extracted from a pool of flies sampled at 2, 4, and $6 \mathrm{~d}$-pi.

Battisti, A. J., Meng, G., Winkler, D. C., McGinnes, L. W., Plevka, P., Steven, A. C., et al. (2012). Structure and assembly of a paramyxovirus matrix protein. Proc. Natl. Acad. Sci. U.S.A. 109, 13996-14000. doi: 10.1073/pnas.1210275109

Boucias, D., Baniszewski, J., Prompiboon, P., Lietze, V., and Geden, C. (2015). Enhancement of the Musca domestica hytrosavirus infection with orally delivered reducing agents. J. Invertebr. Pathol. 124, 35-43. doi: 10.1016/j.jip.2014.10.004

Boucias, D. G., Deng, F., Hu, Z., Garcia-Maruniak, A., and Lietze, V. U. (2013a). Analysis of the structural proteins from the Musca domestica hytrosavirus with an emphasis on the major envelope protein. J. Invertebr. Pathol. 112(Suppl. 1):S44-S52. doi: 10.1016/j.jip.2012.03.016

Boucias, D. G., Kariithi, H. M., Bourtzis, K., Schneider, D. I., Kelley, K., Miller, W. J., et al. (2013b). Trans-generational transmission of the Glossina pallidipes hytrosavirus depends on the presence of a functional symbiome. PLOS ONE 8:e61150. doi: 10.1371/journal.pone.0061150

Brucker, R. M., Funkhouser, L. J., Setia, S., Pauly, R., and Bordenstein, S. R. (2012). Insect Innate Immunity Database (IIID): an annotation tool for identifying immune genes in insect genomes. PLOS ONE 7:e45125. doi: 10.1371 /journal.pone.0045125

Calnan, D. R., and Brunet, A. (2008). The FoxO code. Oncogene 27, 2276-2288. doi: 10.1038/onc.2008.21

Chen, H., Chen, K., Yao, Q., Guo, Z., and Wang, L. (2007). Characterization of a late gene, ORF67 from Bombyx mori nucleopolyhedrovirus. FEBS. Lett. 581, 5836-5842. doi: 10.1016/j.febslet.2007.11.059

Chiramel, A. I., Brady, N. R., and Bartenschlager, R. (2013). Divergent roles of autophagy in virus infection. Cells 2, 83-104. doi: 10.3390/cells20 10083

Choi, K. H. (2012). "Viral polymerases," in Viral Molecular Machines, eds M. G. Rossmann, V. B. Rao (New York, NY: Springer US), 267-304. doi: 10.1007/978-1-4614-0980-9_12

Conesa, A., Götz, S., García-Gómez, J. M., Teról, J., Talon, M., and Robles, M. (2005). Blast2GO: a universal tool for annotation, visualization and analysis in functional genomics research. Bioinformatics 21, 3674-3676. doi: 10.1093/bioinformatics/bti610

Crook, N. E., Clem, R. J., and Miller, L. K. (1993). An apoptosis-inhibiting baculovirus gene with a zinc finger-like motif. J. Virol. 67, 2168-2174.

Decroly, E., Ferron, F., Lescar, J., and Canard, B. (2012). Conventional and unconventional mechanisms for capping viral mRNA. Nat. Rev. Microbiol. 10, 51-65. doi: $10.1038 /$ nrmicro 2675

Ding, S. W. (2010). RNA-based antiviral immunity. Nat. Rev. Immunol. 10, 632-644. doi: 10.1038/nri2824 
Dong, S. Z., Ye, G. Y., Guo, J. Y., and Hu, C. (2009). Roles of ecdysteroid and juvenile hormone in vitellogenesis in an endoparasitic wasp, Pteromalus puparum (Hymenoptera: Pteromalidae). Gen. Comp. Endocr. 160, 102-108. doi: 10.1016/j.ygcen.2008.11.007

Edgar, R., Domrachev, M., and Lash, A. E. (2002). Gene expression omnibus: NCBI gene expression and hybridization array data repository. Nucleic Acids Res. 30, 207-210. doi: 10.1093/nar/30.1.207

Eijkelenboom, A., and Burgering, B. M. (2013). FOXOs: signalling integrators for homeostasis maintenance. Nat. Rev. Mol. Cell Biol. 14, 83-97. doi: $10.1038 / \mathrm{nrm} 3507$

Fay, N., and Panté, N. (2015). Nuclear entry of DNA viruses. Front. Microbiol. 6:467. doi: 10.3389/fmicb.2015.00467

Finn, R. D., Tate, J., Mistry, J., Coggill, P. C., Sammut, S. J., Hotz, H. R., et al. (2008). The Pfam protein families database. Nucl. Acids Res. 36, D281-D288. doi: 10.1093/nar/gkm960

Fischer, U., Jänicke, R. U., and Schulze-Osthoff, K. (2003). Many cuts to ruin: a comprehensive update of caspase substrates. Cell Death Differ. 10, 76-100. doi: $10.1038 /$ si.cdd. 4401160

Garcia-Maruniak, A., Abd-Alla, A. M. M., Salem, T. Z., Parker, A. G., van Oers, M. M., Maruniak, J. E., et al. (2009). Two viruses that cause salivary gland hypertrophy in Glossina pallidipes and Musca domestica are related and form a distinct phylogenetic clade. J. Gen. Virol. 90, 334-346. doi: 10.1099/vir.0.006783-0

Garcia-Maruniak, A., Maruniak, J. E., Farmerie, W., and Boucias, D. G. (2008). Sequence analysis of a non-classified, non-occluded DNA virus that causes salivary gland hypertrophy of Musca domestica, MdSGHV. Virology 377, 184-196. doi: 10.1016/j.virol.2008.04.010

Gilbert, L. I. (2004). Halloween genes encode P450 enzymes that mediate steroid hormone biosynthesis in Drosophila melanogaster. Mol. Cell. Endocrinol. 215, 1-10. doi: 10.1016/j.mce.2003.11.003

Herbert, K. M., and Nag, A. (2016). A tale of two RNAs during viral infection: how viruses antagonize mRNAs and small non-coding RNAs in the host cell. Viruses 8:154. doi: 10.3390/v8060154

Hultmark, D. (1998). "Quantification of antimicrobial activity, using the inhibition-zone assay," in Techniques in Insect Immunology, eds A. Wiesner, G. B. Dunphy, V. J. Maramas, I. Morishima, M. Sugumaran, and M. Yamakawa (Fair Haven: NJ: SOS Publications), 103-107.

Iga, M., and Kataoka, H. (2012). Recent studies on insect hormone metabolic pathways mediated by cytochrome P450 enzymes. Biol. Pharm. Bull. 35, 838-843. doi: $10.1248 /$ bpb.35.838

Jin, J., Dong, W., and Guarino, L. A. (1998). The LEF-4 subunit of baculovirus RNA polymerase has RNA 5'-triphosphatase and ATPase activities. J. Virol. 72, 10011-10019.

Jindra, M. (2016). Something "hairy" in juvenile hormone signaling for mosquito reproduction. Proc. Natl. Acad. Sci U.S.A. 113, 1474-1476. doi: 10.1073/pnas.1524895113

Jindra, M., Palli, S. R., and Riddiford, L. M. (2013). The juvenile hormone signaling pathway in insect development. Annu. Rev. Entomol. 58, 181-204. doi: 10.1146/annurev-ento-120811153700

Kanehisa, M., Goto, S., Kawashima, S., Okuno, Y., and Hattori, M. (2004). The KEGG resource for deciphering the genome. Nucleic Acids Res. 32, D277-D280. doi: 10.1093/nar/gkh063

Kariithi, H. M., van Oers, M. M., Vlak, J. M., Vreysen, M. J., Parker, A. G., and Abd-Alla, A. M. (2013). Virology, epidemiology and pathology of Glossina hytrosavirus, and its control prospects in laboratory colonies of the tsetse fly, Glossina pallidipes (Diptera; Glossinidae). Insects 4, 287-319. doi: 10.3390/insects4030287

Katsuma, S., Mita, K., and Shimada, T. (2007). ERK- and JNK-dependent signaling pathways contribute to Bombyx mori nucleopolyhedrovirus infection. J. Virol. 81, 13700-13709. doi: 10.1128/JVI.01683-07

Kingsolver, M. B., Huang, Z., and Hardy, R. W. (2013). Insect antiviral innate immunity: pathways, effectors, and connections. J. Mol. Biol. 425, 4921-4936. doi: 10.1016/j.jmb.2013.10.006

Konopova, B., and Jindra, M. (2007). Juvenile hormone resistance gene Methoprene-tolerant controls entry into metamorphosis in the beetle Tribolium castaneum. Proc. Natl. Acad. Sci. U.S.A. 104, 10488-10493. doi: $10.1073 /$ pnas.0703719104
Langmead, B., and Salzberg, S. L. (2012). Fast gapped-read alignment with Bowtie 2. Nat. Methods 9, 357-359. doi: 10.1038/nmeth.1923

Levashina, E. A., Moita, L. F., Blandin, S., Vriend, G., Lagueux, M., and Kafatos, F. C. (2001). Conserved role of a complement-like protein in phagocytosis revealed by dsRNA knockout in cultured cells of the mosquito, Anopheles gambiae. Cell 104, 709-718. doi: 10.1016/S0092-8674(01)00267-7

Lietze, V. U., Abd-Alla, A. M. M., and Boucias, D. G. (2011a). Two hytrosaviruses, MdSGHV and GpSGHV, induce distinct cytopathologies in their respective host insects. J. Invertebr. Pathol. 107, 161-163. doi: 10.1016/j.jip.2011.03.006

Lietze, V. U., Geden, C. C., and Boucias, D. G. (2008). "'Here's spitting at you, kid' - oral transmission of the Musca domestica salivary gland hypertrophy virus (MdSGHV) via salivary secretions," in Society Invertebrate Pathology, 41st Annual Meeting of SIP (Coventry: University of Warwick), 120.

Lietze, V. U., Geden, C. J., Blackburn, P., and Boucias, D. G. (2007). Effects of salivary gland hypertrophy virus on the reproductive behavior of the housefly, Musca domestica. Appl. Environ. Microbiol. 73, 6811-6818. doi: 10.1128/AEM.02694-06

Lietze, V. U., Keesling, J. E., Lee, J. A., Vallejo, C. R., Geden, C. J., and Boucias, D. G. (2013). Muscavirus (MdSGHV) disease dynamics in house fly populations: how is this virus transmitted and has it potential as a biological control agent? J. Invertebr. Pathol. 112(Suppl. 1):S40-S43. doi: 10.1016/j.jip.2012.07.017

Lietze, V. U., Salem, T. Z., Prompiboon, P., and Boucias, D. G. (2011b). Tissue tropism of the Musca domestica salivary gland hypertrophy virus. Virus Res. 155, 20-27. doi: 10.1016/j.virusres.2010.06.015

Lietze, V. U., Sims, K. R., Salem, T. Z., Geden, C. J., and Boucias, D. G. (2009). Transmission of MdSGHV among adult house flies, Musca domestica (Diptera: Muscidae), occurs via oral secretions and excreta. J. Invertebr. Pathol. 101, 49-55. doi: 10.1016/j.jip.2009.02.007

Liu, S. W., Katsafanas, G. C., Liu, R., Wyatt, L. S., and Moss, B. (2015). Poxvirus decapping enzymes enhance virulence by preventing the accumulation of dsRNA and the induction of innate antiviral responses. Cell Host Microbe 17, 320-331. doi: 10.1016/j.chom.2015.02.002

Livak, K. J., and Schmittgen, T. D. (2001). Analysis of relative gene expression data using real-time quantitative PCR and the $2^{-\Delta \Delta C_{\mathrm{T}}}$ method. Methods 25, 402-408. doi: 10.1006/meth.2001.1262

Loher, W., and Huber, F. (1966). Nervous and endocrine control of sexual behaviour in a grasshopper (Gomphocerus rufus L., Acridinae). Symp. Soc. Exp. Biol. 20, 381-400.

Marchler-Bauer, A., Derbyshire, M. K., Gonzales, N. R., Lu, S., Chitsaz, F., Geer, L. Y., et al. (2015). CDD: NCBI's conserved domain database. Nucleic Acids Res. 43, D222-D226. doi: 10.1093/nar/gku1221

Martin, M. (2011). Cutadapt removes adapter sequences from high-throughput sequencing reads. EMBnet. J. 17, 10. doi: 10.14806/ej.17.1.200

Miele, S. A., Garavaglia, M. J., Belaich, M. N., and Ghiringhelli, P. D. (2011). Baculovirus: molecular insights on their diversity and conservation. Int. J. Evol. Biol. 2011:379424. doi: 10.4061/2011/379424

Monné, M., Robinson, A. J., Boes, C., Harbour, M. E., Fearnley, I. M., and Kunji, E. R. (2007). The mimivirus genome encodes a mitochondrial carrier that transports dATP and dTTP. J. Virol. 81, 3181-3186. doi: 10.1128/JVI.02386-06

Moreira, L. A., Iturbe-Ormaetxe, I., Jeffery, J. A., Lu, G., Pyke, A. T., Hedges, L. M., et al. (2009). A Wolbachia symbiont in Aedes aegypti limits infection with Dengue, Chikungunya, and Plasmodium. Cell 139, 1268-1278. doi: 10.1016/j.cell.2009.11.042

Mühlbauer, D., Dzieciolowski, J., Hardt, M., Hocke, A., Schierhorn, K. L., Mostafa, A., et al. (2015). Influenza virus-induced caspase-dependent enlargement of nuclear pores promotes nuclear export of viral ribonucleoprotein complexes. J. Virol. 89, 6009-6021. doi: 10.1128/JVI.03531-14

Murvosh, C. M., Fye, R. L., and LaBrecque, G. C. (1964). Studies on the mating behavior of the house fly, Musca domestica L. Ohio J. Sci. 64, 264-271.

Nakahara, K., Kim, K., Sciulli, C., Dowd, S. R., Minden, J. S., and Carthew, R. W. (2005). Targets of microRNA regulation in the Drosophila oocyte proteome. Proc. Natl. Acad. Sci. U.S.A. 102, 12023-12028. doi: 10.1073/pnas.0500053102

Nakamoto, M., Moy, R. H., Xu, J., Bambina, S., Yasunaga, A., Shelly, S. S., et al. (2012). Virus recognition by Toll-7 activates antiviral autophagy in Drosophila. Immunity 36, 658-667. doi: 10.1016/j.immuni.2012.03.003

Narayanan, K., and Makino, S. (2013). Interplay between viruses and host mRNA degradation. Biochim. Biophys. Acta. 1829, 732-741. doi: $10.1016 /$ j.bbagrm.2012.12.003 
Nobiron, I., O’Reilly, D. R., and Olszewski, J. A. (2003). Autographa californica nucleopolyhedrovirus infection of Spodoptera frugiperda cells: a global analysis of host gene regulation during infection, using a differential display approach. J. Gen. Virol. 84, 3029-3039. doi: 10.1099/vir.0.19270-0

Noriega, F. G. (2014). Juvenile hormone biosynthesis in insects: what is new, what do we know, and what questions remain? Int. Sch. Res. Notices 2014:967361 doi: $10.1155 / 2014 / 967361$

Okamura, K., Ishizuka, A., Siomi, H., and Siomi, M. C. (2004). Distinct roles for Argonaute proteins in small RNA-directed RNA cleavage pathways. Genes Dev. 18, 1655-1666. doi: 10.1101/gad.1210204

Ono, H., Rewitz, K. F., Shinoda, T., Itoyama, K., Petryk, A., Rybczynski, R., et al. (2006). Spook and Spookier code for stage-specific components of the ecdysone biosynthetic pathway in Diptera. Dev. Biol. 298, 555-570. doi: 10.1016/j.ydbio.2006.07.023

Paradkar, P. N., Trinidad, L., Voysey, R., Duchemin, J. B., and Walker, P. J. (2012). Secreted Vago restricts West Nile virus infection in Culex mosquito cells by activating the Jak-STAT pathway. Proc. Natl. Acad. Sci. U.S.A. 109, 18915-18920. doi: 10.1073/pnas.1205231109

Peferoen, M., and de Loof, A. (1986). Synthesis of vitellogenic and nonvitellogenic yolk proteins by the fat body and the ovary of Leptinotarsa decemlineata. Comp. Biochem. Physiol. B Biochem. Mol. Biol. 83B, 251-254. doi: 10.1016/0305-0491(86)90362-7

Prompiboon, P., Lietze, V.-U., Denton, J. S. S., Geden, C. J., Steenberg, T., and Boucias, D. G. (2010). Musca domestica salivary gland hypertrophy virus, a globally distributed insect virus that infects and sterilizes female houseflies. Appl. Environ. Microbiol. 76, 994-998. doi: 10.1128/AEM.02424-09

Rewitz, K. F., Rybczynski, R., Warren, J. T., and Gilbert, L. I. (2006). The Halloween genes code for cytochrome P450 enzymes mediating synthesis of the insect moulting hormone. Biochem. Soc. Trans. 34, 1256-1260. doi: 10.1042/BST0341256

Royet, J., Gupta, D., and Dziarski, R. (2011). Peptidoglycan recognition proteins: modulators of the microbiome and inflammation. Nat. Rev. Immunol. 11, 837-851. doi: 10.1038/nri3089

Salem, T. Z., Garcia-Maruniak, A., Lietze, V. U., Maruniak, J. E., and Boucias, D. G. (2009). Analysis of transcripts from predicted open reading frames of Musca domestica salivary gland hypertrophy virus. J. Gen. Virol. 90, 1270-1280. doi: 10.1099/vir.0.009613-0

Schwenke, R. A., Lazzaro, B. P., and Wolfner, M. F. (2016). Reproduction: immunity trade-offs in insects. Annu. Rev. Entomol. 61, 239-256. doi: 10.1146/annurev-ento-010715-023924

Scott, J. G., Warren, W. C., Beukeboom, L. W., Bopp, D., Clark, A. G., Giers, S. D., et al. (2014). Genome of the house fly, Musca domestica L., a global vector of diseases with adaptations to a septic environment. Genome Biol. 15:466. doi: 10.1186/s13059-014-0466-3

Shelly, S., Lukinova, N., Bambina, S., Berman, A., and Cherry, S. (2009). Autophagy is an essential component of Drosophila immunity against vesicular stomatitis virus. Immunity 30, 588-598. doi: 10.1016/j.immuni.2009.02.009

Shoji-Kawata, S., and Levine, B. (2009). Autophagy, antiviral immunity, and viral countermeasures. BBA Mol. Cell Res. 1793, 1478-1484. doi: 10.1016/j.bbamcr.2009.02.008

Siegenthaler, C., Maroy, P., Hediger, M., Dubendorfer, A., and Bopp, D. (2010). Hormones and sex-specific transcription factors jointly control yolk protein synthesis in Musca domestica. Int. J. Evol. Biol. 2010:291236. doi: $10.4061 / 2009 / 291236$
Song, J., Guo, W., Jiang, F., Kang, L., and Zhou, S. (2013). Argonaute 1 is indispensable for juvenile hormone mediated oogenesis in the migratory locust, Locusta migratoria. Insect Biochem. Mol. Biol. 43, 879-887. doi: 10.1016/j.ibmb.2013.06.004

Song, J., Wang, X., Hou, D., Huang, H., Liu, X., Deng, F., et al. (2016). The host specificities of baculovirus per os infectivity factors. PLoS ONE 11:e0159862. doi: 10.1371/journal.pone.0159862

Teal, P. E., Jones, D., Jones, G., Torto, B., Nyasembe, V., Borgemeister, C., et al. (2014). Identification of methyl farnesoate from the hemolymph of insects. J. Nat. Prod. 77, 402-405. doi: 10.1021/np400807v

Thomson, B. J. (2001). Viruses and apoptosis. Int. J. Exp. Pathol. 82, 65-76. doi: 10.1111/j.1365-2613.2001.iep195.x

Untergasser, A., Nijveen, H., Rao, X., Bisseling, T., Geurts, R., and Leunissen, J. A. (2007). Primer3Plus, an enhanced web interface to Primer3. Nucleic Acids Res. 35, W71-W74. doi: 10.1093/nar/gkm306

Van Attikum, H., and Gasser, S. M. (2009). Crosstalk between histone modifications during the DNA damage response. Trends Cell Biol. 19, 207-217. doi: 10.1016/j.tcb.2009.03.001

Vigdorovich, V., Miller, A. D., and Strong, R. K. (2007). Ability of hyaluronidase 2 to degrade extracellular hyaluronan is not required for its function as a receptor for jaagsiekte sheep retrovirus. J. Virol. 81, 3124-3129. doi: 10.1128/JVI.02177-06

Wang, Y., Kleespies, R. G., Huger, A. M., and Jehle, J. A. (2007). The genome of Gryllus bimaculatus nudivirus indicates an ancient diversification of baculovirus-related nonoccluded nudiviruses of insects. J. Virol. 81, 5395-5406. doi: 10.1128/JVI.02781-06

Weitzman, M. D., Lilley, C. E., and Chaurushiya, M. S. (2010). Genomes in conflict: maintaining genome integrity during virus infection. Annu. Rev. Microbiol. 64, 61-81. doi: 10.1146/annurev.micro.112408.134016

Yano, T., Mita, S., Ohmori, H., Oshima, Y., Fujimoto, Y., Ueda, R., et al. (2008). Autophagic control of listeria through intracellular innate immune recognition in Drosophila. Nat. Immunol. 9, 908-916. doi: 10.1038/ni.1634

Yao, J. Q., and Yu, F. (2011). DEB: A web interface for RNA-seq digital gene expression analysis. Bioinformation 7, 44-45. doi: 10.6026/973206300 07044

Yordy, B., Tal, M. C., Hayashi, K., Arojo, O., and Iwasaki, A. (2013). Autophagy and selective deployment of Atg proteins in antiviral defense. Int. Immunol. 25, 1-10. doi: 10.1093/intimm/dxs101

Zou, Z., Saha, T. T., Roy, S., Shin, S. W., Backman, T. W., Girke, T., et al. (2013). Juvenile hormone and its receptor, methoprene-tolerant, control the dynamics of mosquito gene expression. Proc. Natl. Acad. Sci. U.S.A. 110, E2173-E2181. doi: 10.1073/pnas.1305293110

Conflict of Interest Statement: The authors declare that the research was conducted in the absence of any commercial or financial relationships that could be construed as a potential conflict of interest.

Copyright (C) 2017 Kariithi, Yao, Yu, Teal, Verhoeven and Boucias. This is an openaccess article distributed under the terms of the Creative Commons Attribution License (CC BY). The use, distribution or reproduction in other forums is permitted, provided the original author(s) or licensor are credited and that the original publication in this journal is cited, in accordance with accepted academic practice. No use, distribution or reproduction is permitted which does not comply with these terms. 